\title{
A Study of Knowledge, Attitude and Practice Relative to Tuberculosis among Korean Adults; 1970
}

\author{
Nak Chin Chung, M.D., M.P.H.
}

Ministry of Health and Social Affairs, Republic of Korea

\section{INTRODUCTION}

Tuberculosis is a serious problem in Korea. It is estimated, on the basis of the results of the first prevalence survey in 1965, that there are about 1.25 million tuberculosis sufferers or $5.1 \%$ of the population aged 5 years and above. Of this number, 226, 000 were infectious and no less than 20,000 of them were expected to die from this disease each year. A great majority of patients and those who die from tuberculosis are at their productive ages (i.e. 20 50 years of age). Thus, this disease is a great obstacle to industrialization and economic growth of the nation. In addition, the disease causes a socio-pathologic phenomenon resulting from broken homes and other undesirable situations among the families of the tuberculosis patients.

The national tuberculosis program has been in operation since 1962. It started with very meager facilities and very few trained staff members. It has developed gradually to the present status with service being extended to the whole country through the health center and subcenter network, enlisting the participation of more than 2,000 full-time workers.

Since 1966 the Korean National Tuberculosis Association (KNTA) and the Ministry of Health and Social Affairs (MHSA) have conducted an active tuberculosis health education program. It was assumed that the educational program achieved its objectives in promoting the public's understanding of tuberculosis, but there was no data to support this assumption. We did not know whether the program was meeting the needs of the people or whether they had a clear understanding of the disease or the service the government renders in tuberculosis control. Moreover, we did not know how acceptable they found the service was both organizations recognized the fundamental need to obtain this information in order to implement an effectively revised health education program. Therefore, when the second national tuberculosis prevalence survey was conducted in 1970, questions were asked to a selected group of respondents for the purpose of determining:

1. the knowledge, attitude and practice concerning tuberculosis;

2. how much was understood about the free public health service in tuberculosis control;

3. the degree of acceptability of the tuberculosis control program.

Data collected in this survey is to serve as the basis of developing a stronger tuberculosis control program, especially in its educational aspects; so that better service can be offered.

\section{METHODOLOGY}

\section{1) Sampling}

For the re-appraisal of the tuberculosis problem, the KNTA and MHSA conducted the second nationwide tuberculosis prevalence survey during the period of April 17 July 31, 1970. The main objectives of the survey were to estimate the total number of tuberculosis patients and the prevalence of tuberculosis infection in the population. A statistical multistage probability sample was selected in proportion to the number of households in 86 sample areas, of which 46 were urban and 40 were rural, representing the total population. For the health education survey we sampled every 10th subject listed for the prevalence survey in the 86 areas. These 86 sampling areas were distributed over two special cities, Seoul and Pusan,

Reported to 7th IUAT of Eastern Region on the date Nov. 21, 1971 
and nine provinces of Korea. Samples drawn were of both sexes aged 20 and above.

\section{2) Interviewing}

The total for the health education survey consists of 971 persons drawn from the 9,707 persons selected for the prevalence survey. Each sample was drawn when the census investigation was carried out to confirm the actual number of subjects living in the area at that time. The census survey was carried out three days prior to the prevalence survey. From the 971 samples, 826 interviews were completed ( 85 $\%)$. Only 17 persons refused to be interviewed ( 0.2 $\%)$ while 128 persons (13.2\%) were "never home" at two home visits.

Table 1. Number and Proportion of Total Sample and Persons Interviewed by Age Group

\begin{tabular}{c|r|r|r}
\hline \hline Age group & $\begin{array}{c}\text { Persons to be } \\
\text { interviewed } \\
\text { (a) }\end{array}$ & $\begin{array}{c}\text { Persons } \\
\text { interviewed } \\
\text { (b) }\end{array}$ & $\begin{array}{c}\text { Per cent } \\
\frac{\mathrm{b}}{\mathrm{a}}\end{array}$ \\
\hline $20 \sim 29$ & 278 & 195 & 70.1 \\
$30 \sim 39$ & 280 & 263 & 93.9 \\
$40 \sim 49$ & 183 & 152 & 83.1 \\
$50 \sim 59$ & 122 & 119 & 97.5 \\
$60+$ & 108 & 97 & 89.9 \\
\hline Total & 971 & 826 & 85.1 \\
\hline
\end{tabular}

The average interview required about 17 minutes.

Those whom the interviews could not contact were listed and reasons given. To arouse the cooperation of people to participate in the prevalence survey, the health education campaign was conducted immediately after the census investigation. Samples that could not be made by that time were discarded.

\section{3) The Questionnaire}

The questionnaire used included information pertaining to the following categories:

a) Background characteristics; demographic items such as AGE, SEX, EDUCATION, LITERACY, OCCUPATION, and economic status and residence. Also included were availability of newspaper and radio, place of treatment for illnesses, etc.

b) Knowledge of the disease, tuberculosis.

c) Understanding of the nature of tuberculosis; mode of transmission, symptoms and signs, susceptitbility and curability.

d) Information source of tuberculosis. e) Experience of tuberculosis among family members.

f) Understanding of the diagnostic measures of tuberculosis.

g) Experience of $x$-ray and sputum examination and acceptability of free mobile $\mathrm{x}$-ray examination.

h) Attitudes towards the place of treatment of tuberculosis, knowledge of available free treatment of available free treatment of tuberculosis.

i) Knowledge and acceptability of BCG vaccinaiton.

j) Experience in utilizing health center services.

\section{ANALYSIS AND STUDY OF THE FINDINGS}

\section{Background Characteristics of the Samples}

\section{Residence}

Among the 826 interviews completed, 361 (43.7\%) were from the urban and the remaining 465 (56.3\%) were from the rural areas. Those who had been living at their present addresses for more than three years were 67.5 percent of the total respondents; 56.0 per cent in the urban and 67.5 per cent in the rural (Table 2) areas.

Table 2. Number and Per Cent of Urban and Rural Respondents by Length of Residence at Present Address

\begin{tabular}{|c|c|c|c|c|c|c|}
\hline \multirow{2}{*}{$\begin{array}{l}\text { Years at } \\
\text { present } \\
\text { address }\end{array}$} & \multicolumn{2}{|c|}{ Urban } & \multicolumn{2}{|c|}{ Rural } & \multicolumn{2}{|c|}{ Total } \\
\hline & No. & $\%$ & No. & $\%$ & No. & $\%$ \\
\hline Less than 1 & 98 & 27.1 & 67 & 14.6 & 165 & 19.1 \\
\hline $1 \sim 2$ & 27 & 7.5 & 16 & 3.4 & 43 & 5.2 \\
\hline $2 \sim 3$ & 34 & 9.4 & 26 & 5.6 & 60 & 7.2 \\
\hline $3+$ & 202 & 56.0 & 356 & 76.3 & 558 & 67.5 \\
\hline Total & 361 & 100.0 & 465 & 100.0 & 826 & 100.0 \\
\hline
\end{tabular}

Population migration to escape socio-economic hardship originating from regional differences is a general trend. Population movement between urban and rural and within the urban areas has been frequent. Such a population movement creates vexing problems which affect proper follow-up of the patients under control. When a patient moves to other areas, he usually becomes "lost" and is probably registered as a new patient again at the health center of his new jurisdiction. To meet frequent changes of address, a 
follow-up system must be established to keep infectious cases under proper management by utilizing citizen registration certificates which were established in 1969 for the whole population over age of 18 .

\section{Distribution of Sex}

The proportion of female samples were greater than that of males. This was due to the fact that more male samples were unable to be contacted and considerable number of young men had been drafted into military service.

Table 3. Number and Per Cent of Urban and Rural Respondent by Sex

\begin{tabular}{l|r|r|r|r|r|r}
\hline \multirow{2}{*}{ Sex } & \multicolumn{2}{|c|}{ Urban } & \multicolumn{2}{c|}{ Rural } & \multicolumn{2}{c}{ Total } \\
\cline { 2 - 6 } \cline { 3 - 7 } & No. & $\%$ & No. & $\%$ & No. & $\%$ \\
\hline Male & 175 & 48.5 & 217 & 47.0 & 392 & 47.5 \\
Female & 186 & 51.5 & 245 & 53.0 & 434 & 52.5 \\
\hline Total & 361 & 100.0 & 462 & 100.0 & 826 & 100.0 \\
\hline
\end{tabular}

\section{Age Distribution}

The proportion of young respondents under 30 years of age was smaller than that of samples for the prevalence survey $(23.6 \%$ vs. $28.6 \%)$. This was again due to the fact that a considerable number of samples were not at home during the day of the interview, particularly men.

Table 4. Number and Per Cent of Urban and Rural Respondents by Age Group

\begin{tabular}{c|r|r|r|r|r|r}
\hline \hline \multirow{2}{*}{ Age group } & \multicolumn{2}{|c|}{ Urban } & \multicolumn{2}{c|}{ Rural } & \multicolumn{2}{c}{ Total } \\
\cline { 2 - 3 } \cline { 5 - 7 } & No. & $\%$ & No. & $\%$ & No. & $\%$ \\
\hline $20 \sim 29$ & 91 & 25.2 & 104 & 22.4 & 195 & 23.6 \\
$30 \sim 39$ & 128 & 35.5 & 135 & 29.0 & 263 & 31.8 \\
$40 \sim 49$ & 65 & 18.0 & 87 & 18.7 & 152 & 18.4 \\
$50 \sim 59$ & 41 & 11.3 & 78 & 16.8 & 119 & 14.4 \\
$60+$ & 36 & 10.0 & 61 & 13.1 & 97 & 11.8 \\
\hline Total & 361 & 100.0 & 465 & 100.0 & 826 & 100.0 \\
\hline
\end{tabular}

\section{Educational Level}

The illiterate rate of the respondents was 17.8 per cent. The proportion with no schooling comprised 31.5 per cent of the total respondents, whereas 34.6 per cent finished the middle school or above. A marked difference was observed between urban and rural areas in the group with no school education; 16.6
결핵 및 호홉기질환 Vol. 18, No. 4, October, 1971

per cent in the urban and 43.0 per cent in the rural areas.

Table 5. Number and Per Cent of Urban and Rural Respondents by Educational Level

\begin{tabular}{l|r|r|r|r|r|r}
\hline \hline \multirow{2}{*}{$\begin{array}{l}\text { Educational } \\
\text { levels }\end{array}$} & \multicolumn{2}{|c|}{ Urban } & \multicolumn{2}{c|}{ Rural } & \multicolumn{2}{c}{ Total } \\
\cline { 2 - 5 } \cline { 5 - 7 } & No. & \multicolumn{1}{c|}{$\%$} & No. & \multicolumn{1}{c}{$\%$} & No. & $\%$ \\
\hline Illiterate & 29 & 8.0 & 118 & 25.4 & 147 & 17.8 \\
$\begin{array}{l}\text { Literate no } \\
\text { schooling }\end{array}$ & 31 & 8.6 & 82 & 17.6 & 113 & 13.7 \\
$\begin{array}{l}\text { Elementary } \\
\text { school }\end{array}$ & 103 & 28.5 & 177 & 38.1 & 280 & 33.9 \\
$\begin{array}{l}\text { Middle } \\
\text { school }\end{array}$ & 77 & 21.3 & 48 & 10.3 & 125 & 15.1 \\
$\begin{array}{l}\text { High school } \\
\text { College }\end{array}$ & 89 & 24.7 & 32 & 6.9 & 121 & 14.7 \\
\hline Total & 32 & 8.9 & 8 & 1.7 & 40 & 4.8 \\
\hline
\end{tabular}

Occupation

For convenience, similar types of occupations were combined into one category, such as professional workers, managerial workers and college students. The Economic Board statistical data for 1968 show the following:

\section{Category}

Farmers, laborers

Clerical

Professional, managerial
Sales workers

\section{$\%$ of Population} $52 \%$

13.4

3.9
Since housewives (in Korea housewife means wives or mothers of a home) have an important role at home, they were classified in a separate category.

Table 6. Number and Per Cent of Urban and Rural Respondents by Occupation

\begin{tabular}{l|r|r|r|r|r|r}
\hline \hline \multirow{2}{*}{ Occupation } & \multicolumn{2}{|c|}{ Urban } & \multicolumn{2}{|c|}{ Rural } & \multicolumn{2}{c}{ Total } \\
\cline { 2 - 6 } \cline { 5 - 7 } & No. & \multicolumn{1}{c}{$\%$} & No. & $\%$ & No. & $\%$ \\
\hline Laborer & 122 & 33.8 & 82 & 17.6 & 204 & 24.7 \\
Farmer & 29 & 8.0 & 102 & 21.9 & 131 & 15.9 \\
Housewife & 89 & 24.7 & 188 & 40.4 & 277 & 33.5 \\
$\begin{array}{c}\text { Sales } \\
\text { worker }\end{array}$ & 61 & 16.9 & 31 & 6.6 & 92 & 11.1 \\
$\begin{array}{c}\text { Technical } \\
\text { worker }\end{array}$ & 21 & 6.1 & 9 & 1.9 & 31 & 3.8 \\
$\begin{array}{c}\text { Clerical } \\
\text { worker }\end{array}$ & 14 & 3.9 & 28 & 6.1 & 42 & 5.1 \\
professional \\
worker
\end{tabular}




\section{Economic Condition}

It was difficult to estimate accurately the economic conditions only through simple interview, particularly in rural areas. The author has the impression that there is no distinctive difference between middle and upper socio-economic classes in educational levels. The lower classes might be comparable between the urban and the rural, but not for middle and upper socio-economic classes.

Table 7. Number and Per Cent of Urban and Rural Respondents by Economic Condition

\begin{tabular}{l|r|r|r|r|r|r}
\hline \multirow{2}{*}{$\begin{array}{l}\text { Economic } \\
\text { condition }\end{array}$} & \multicolumn{2}{|c|}{ Urban } & \multicolumn{2}{c|}{ Rural } & \multicolumn{2}{c}{ Total } \\
\cline { 2 - 6 } & No. & $\%$ & No. & $\%$ & No. & $\%$ \\
\hline Upper & 46 & 12.7 & 29 & 6.4 & 75 & 9.2 \\
Middle & 209 & 57.8 & 185 & 39.7 & 394 & 47.6 \\
Lower & 106 & 29.5 & 251 & 53.9 & 357 & 43.2 \\
\hline Tota1 & 361 & 100.0 & 465 & 100.0 & 826 & 100.0 \\
\hline
\end{tabular}

\section{Exposure to Mass Media}

It is encouraging to note that 43.8 per cent of the respondents read newspapers and 77 per cent of them have radios or amplifier network systems. In a rural village, those who cannot afford to buy radios usually have amplifier network systems rented by a private amplifier station for a small charge. As to radio communication, it became obvious that the respondents overwhelmingly listen to the radio which appears to be a most viable means of communication, particularly for the illiterate. If such mass media, newspapers and radio broadcasts, are properly and efficiently utilized for health education of public health programs, the effectiveness of the programs can be truly improved.

Table 8. Number and Per Cent of Urban and Rural Respondents by Use of Radio and Newspaper

\begin{tabular}{l|r|c|c|c|c|c}
\hline \multirow{2}{*}{ Mass media } & \multicolumn{2}{|c|}{ Urban } & \multicolumn{2}{c|}{ Rural } & \multicolumn{2}{c}{ Total } \\
\cline { 2 - 6 } & No. & $\%$ & No. & $\%$ & No. & $\%$ \\
\hline Radio & 308 & 85.3 & 329 & 70.7 & 637 & 77.1 \\
Newspaper & 207 & 57.4 & 155 & 33.3 & 362 & 43.8 \\
\hline (Total & \multicolumn{2}{|c|}{$(316)$} & \multicolumn{2}{|c|}{ (465) } & \multicolumn{2}{|c}{ (826) } \\
\hline
\end{tabular}

Table 9. Number and Per Cent of Respondents according to Economic Condition by Use of Radio and Newspaper

\begin{tabular}{|c|c|c|c|c|c|c|c|c|}
\hline \multirow{3}{*}{ Mass media } & \multicolumn{8}{|c|}{ Eccnomic conditions } \\
\hline & \multicolumn{2}{|c|}{ Upper } & \multicolumn{2}{|c|}{ Middle } & \multicolumn{2}{|c|}{ Lower } & \multicolumn{2}{|c|}{ Total } \\
\hline & No. & $\%$ & No. & $\%$ & No & $\%$ & No. & $\%$ \\
\hline Radio & 75 & 100.0 & 339 & 86.0 & 223 & 62.2 & & 77.1 \\
\hline Newspaper & 65 & 86.0 & 220 & 55.8 & & 21.5 & 362 & 43.8 \\
\hline $\begin{array}{l}\text { (Total } \\
\text { respondents) }\end{array}$ & & (75) & & 94) & & 375) & & 826) \\
\hline
\end{tabular}

\section{Experience of Illness}

Since the survey was carried out for a period of four months, the experience of illness shown in Tables 10 and 11 cannot be statistical value, but the impression of poor health among the respondents can be realized. The difference between various age groups and the difference between urban and rural areas were marked. The experience of various sicknesses during the last one year was $31.2 \%$ in whole area.

Table 10. Number and Per Cent of Respondents who Experienced Illness during the Last One Year by Age Group

\begin{tabular}{l|r|r|r|r|r|r}
\hline \hline \multirow{2}{*}{ Age group } & \multicolumn{2}{|c|}{ Sick } & \multicolumn{2}{c|}{ Not sick } & \multicolumn{2}{c}{ Total } \\
\cline { 2 - 6 } \cline { 5 - 7 } & No. & $\%$ & No. & $\%$ & No. & $\%$ \\
\hline 20 29 & 36 & 18.4 & 159 & 81.6 & 195 & 100.0 \\
$30 \sim 39$ & 73 & 27.7 & 190 & 72.3 & 263 & 100.0 \\
$40 \sim 49$ & 55 & 36.1 & 97 & 63.9 & 152 & 100.0 \\
$50 \sim 59$ & 50 & 42.0 & 69 & 58.0 & 119 & 100.0 \\
$60+$ & 44 & 45.3 & 53 & 54.7 & 97 & 100.0 \\
\hline Total & 258 & 31.2 & 568 & 68.8 & 826 & 100.0 \\
\hline
\end{tabular}

Table 11. Number and Per Cent of Urban and Rural Respondents Who Experienced Illness during the Last One Year

\begin{tabular}{l|r|r|r|r|r|r}
\hline \hline \multirow{2}{*}{ Area } & \multicolumn{2}{|c|}{ Sick } & \multicolumn{2}{c|}{ Not sick } & \multicolumn{2}{c}{ Total } \\
\cline { 2 - 7 } & No. & $\%$ & No. & $\%$ & No. & $\%$ \\
\hline Urban & 88 & 24.4 & 273 & 75.6 & 361 & 100.0 \\
Rural & 170 & 28.8 & 295 & 61.2 & 465 & 100.0 \\
\hline Total & 258 & 31.2 & 568 & 68.8 & 826 & 100.0 \\
\hline
\end{tabular}

\section{Treatment for Illness}

In reply to the question, "From whom do you seek treatment in case you or your family members are 
sick?”, only 35.4 per cent said they went to a medical doctor. Two-thirds of the respondents relied on pharmacists, Chinese herb doctors, and others who could not provide proper treatment. This may have been due to expensive medical charges and a shortage of medical doctors in rural areas. With increased

Table 12. Number and Per Cent of the Urban and Rural Respondents in Treatment of Illness by Medical Personnel

\begin{tabular}{l|r|r|r|r|r|r}
\hline \hline \multirow{2}{*}{$\begin{array}{l}\text { Medical } \\
\text { personnel }\end{array}$} & \multicolumn{2}{|c|}{ Urban } & \multicolumn{2}{c|}{ Rural } & \multicolumn{2}{c}{ Total } \\
\cline { 2 - 5 } \cline { 6 - 7 } & No. & \multicolumn{1}{c}{$\%$} & No. & $\%$ & No. & $\%$ \\
\hline M.D. & 154 & 42.6 & 139 & 29.8 & 293 & 35.4 \\
Pharmacist & 151 & 41.0 & 209 & 44.9 & 360 & 43.5 \\
$\begin{array}{l}\text { Chinese herb } \\
\text { doctor }\end{array}$ & 22 & 6.9 & 52 & 11.2 & 74 & 8.9 \\
$\begin{array}{l}\text { Midwife or } \\
\text { nurse }\end{array}$ & 8 & 2.5 & 11 & 2.4 & 19 & 2.3 \\
$\begin{array}{l}\text { Fortune } \\
\text { teller or } \\
\text { moodang }\end{array}$ & 5 & 1.2 & 25 & 5.4 & 30 & 3.4 \\
$\begin{array}{c}\text { Don't know } \\
\text { Total }\end{array}$ & 21 & 5.8 & 29 & 6.3 & 50 & 6.5 \\
\hline
\end{tabular}

Table 13. Number and Per Cent of the Respondents in Treatment of Illness by Medical Personnel and by Economic Condition

\begin{tabular}{|c|c|c|c|c|c|c|c|c|}
\hline \multirow{3}{*}{$\begin{array}{l}\text { Medical } \\
\text { personnel }\end{array}$} & \multicolumn{8}{|c|}{ Economic condition } \\
\hline & \multicolumn{2}{|c|}{ Upper } & \multicolumn{2}{|c|}{ Middle } & \multicolumn{2}{|c|}{ Lower } & \multicolumn{2}{|c|}{ Total } \\
\hline & No. & $\%$ & No. & $\%$ & No. & $\%$ & No. & $\%$ \\
\hline M. D. & 48 & 64.0 & 148 & 37.6 & 97 & 27.1 & 293 & 35.4 \\
\hline Pharmacist & 20 & 26.7 & 167 & 42.4 & 173 & 48.4 & 360 & 43.5 \\
\hline $\begin{array}{l}\text { Chinese-herb } \\
\text { doctor }\end{array}$ & 4. & 5.4 & 36 & 9.2 & 34 & 9.5 & 74 & 8.9 \\
\hline $\begin{array}{l}\text { Midwife or } \\
\text { nurse }\end{array}$ & 1 & 1.3 & 10 & 2.5 & 8 & 2.2 & 19 & 2.3 \\
\hline $\begin{array}{l}\text { Fortune } \\
\text { teller or } \\
\text { sorceress }\end{array}$ & 1 & 1.3 & 13 & 3.3 & 16 & 4.5 & 30 & 3.4 \\
\hline Don't know & 1 & 1.3 & 20 & 5.0 & 29 & 6.8 & 50 & 6.5 \\
\hline Total & 75 & 100.0 & 394 & 100.0 & 357 & 100.0 & 826 & 100.0 \\
\hline
\end{tabular}

결핵 및 호홉기질환 Vol. 18, No. 4, October, 1971

recognition of scientific medical practices, very few people (3.4\%) seek treatment from fortune tellers or sorceresses.

\section{Knowledge of the Disease, "Tuberculosis"}

"Have you ever heard of the disease, tuberculosis?"

The majority of the respondents heard the disease, "tuberculosis" or know it as a lung disease which has been commonly called by old people. 12.2 per cent of the respondents were still ignorant of this age-old disease. Females, usually lower in educational level than males of the same age groups, had a rate of 16. 1 per cent ignorance of the disease while it was only 8.0 per cent in the males (Table 14,15 ). By age group the highest rate of incorrect answer (26.8\%) was observed among the group of 60 and above and the next of 16.5 per cent in the group of $20 \sim 29$ (Table 16). Over 95 per cent of the groups of high school and college education knew the disease, but the

Number and Per Cent of the Respondent

Table 14. Classification by Urban and Rural

\begin{tabular}{|c|c|c|c|c|c|c|}
\hline \multirow{2}{*}{ Answer } & \multicolumn{2}{|c|}{ Urban } & \multicolumn{2}{|c|}{ Rural } & \multicolumn{2}{|c|}{ Total } \\
\hline & No. & $\%$ & No. & $\%$ & No. & $\%$ \\
\hline know & 335 & 92.8 & 390 & 83.9 & 725 & 87.8 \\
\hline Don't know & 26 & 7.2 & 75 & 16.1 & 101 & 12.2 \\
\hline Total & 361 & 100.0 & 465 & 100.0 & 826 & 100.0 \\
\hline
\end{tabular}

Table 15. Classification by Sex

\begin{tabular}{l|r|r|r|r|r|r}
\hline \hline \multirow{2}{*}{ Answer } & \multicolumn{2}{|c|}{ Male } & \multicolumn{2}{c|}{ Female } & \multicolumn{2}{c}{ Both sexes } \\
\cline { 2 - 6 } & No. & $\%$ & No. & $\%$ & No. & $\%$ \\
\hline Know & 361 & 92.0 & 364 & 83.9 & 725 & 87.8 \\
Don't know & 31 & 8.0 & 70 & 16.1 & 101 & 12.2 \\
\hline Total & 392 & 100.0 & 434 & 100.0 & 826 & 100.0 \\
\hline
\end{tabular}

Table 16. Classification by Age Group

\begin{tabular}{|c|c|c|c|c|c|c|c|c|c|c|c|c|}
\hline \multirow{3}{*}{ Answer } & \multicolumn{12}{|c|}{ Age group } \\
\hline & \multicolumn{2}{|c|}{$20 \sim 29$} & \multicolumn{2}{|c|}{$30 \sim 39$} & \multicolumn{2}{|c|}{$40 \sim 49$} & \multicolumn{2}{|c|}{$50 \sim 59$} & \multicolumn{2}{|c|}{$60+$} & \multicolumn{2}{|c|}{ Total } \\
\hline & No. & $\%$ & No. & $\%$ & No. & $\%$ & No. & $\%$ & No. & $\%$ & No. & $\%$ \\
\hline Know & 163 & 83.5 & 241 & 91.8 & 145 & 95.4 & 105 & 88.0 & 71 & 73.2 & 725 & 87.8 \\
\hline Don't know & 32 & 16.5 & 22 & 8. 2 & 7 & 4.6 & 14 & 12.0 & 26 & 26.8 & 101 & 12.2 \\
\hline Total & 195 & 100.0 & 263 & 100.0 & 152 & 100.0 & 119 & 100.0 & 97 & 100.0 & 826 & 100.0 \\
\hline
\end{tabular}


결핵 및 호흡기질환 Vol. 18, No. 4, October, 1971

Table 17. Classification by Educational Level

\begin{tabular}{|c|c|c|c|c|c|c|c|c|c|c|c|c|c|c|}
\hline \multirow{2}{*}{ Answer } & \multicolumn{2}{|c|}{ Illiterate } & \multicolumn{2}{|c|}{$\begin{array}{l}\text { Literate no } \\
\text { schooling }\end{array}$} & \multicolumn{2}{|c|}{$\begin{array}{c}\text { Elementary } \\
\text { school } \\
\end{array}$} & \multicolumn{2}{|c|}{$\begin{array}{l}\text { Middle } \\
\text { school }\end{array}$} & \multicolumn{2}{|c|}{ High school } & \multicolumn{2}{|c|}{ College } & \multicolumn{2}{|c|}{ Total } \\
\hline & No. & $\%$ & No. & $\%$ & No. & $\%$ & No. & $\%$ & No. & $\%$ & No & $\%$ & No. & $\%$ \\
\hline Know & 65 & 44.2 & 78 & 69.1 & 219 & 78.2 & 108 & 86.1 & 115 & 95.0 & 40 & 100.0 & 725 & 87.8 \\
\hline Don't know & 82 & 55.8 & 35 & 30.9 & 61 & 21.8 & 17 & 13.9 & 6 & 05.0 & - & - & 101 & 12.2 \\
\hline Total & 147 & 100.0 & 113 & 100.0 & 228 & 100.0 & 125 & 100.0 & 121 & 100.0 & 40 & 100.0 & 826 & 100.0 \\
\hline
\end{tabular}

Table 18. Classification by Occupation

\begin{tabular}{|c|c|c|c|c|c|c|c|c|c|c|c|c|c|c|c|c|}
\hline \multirow{2}{*}{ Answer } & \multicolumn{2}{|c|}{ Laborer } & \multicolumn{2}{|c|}{ Farmer } & \multicolumn{2}{|c|}{ Housewife } & \multicolumn{2}{|c|}{$\begin{array}{c}\text { Sales } \\
\text { worker }\end{array}$} & \multicolumn{2}{|c|}{$\begin{array}{c}\text { Technical } \\
\text { worker }\end{array}$} & \multicolumn{2}{|c|}{$\begin{array}{l}\text { Clerical } \\
\text { worker }\end{array}$} & \multicolumn{2}{|c|}{$\begin{array}{c}\text { Professional } \\
\text { worker }\end{array}$} & \multicolumn{2}{|c|}{ Total } \\
\hline & $\%$ & No. & $\%$ & No. & No. & $\%$ & No. & $\%$ & No. & $\%$ & No. & $\%$ & No. & $\%$ & No. & $\%$ \\
\hline Know & 167 & 81.8 & 108 & 82.2 & 252 & 91.1 & 81 & 88.5 & 29 & 93.5 & 40 & 95.2 & 48 & 98.0 & 725 & 87.7 \\
\hline Don't know & 37 & 18.2 & 23 & 17.8 & 25 & 8.9 & 11 & 11.5 & 2 & 6.5 & 2 & 4.8 & 1 & 2.0 & 101 & 12.3 \\
\hline Total & 204 & 100.0 & 131 & 100.0 & 277 & 100.0 & 92 & 100.0 & 31 & 100.0 & 42 & 100.0 & 49 & 100.0 & 826 & 100.0 \\
\hline
\end{tabular}

disease, but the groups of illiterate and literate without schooling never heard of the disease in 55.8 per cent and 30.9 per cent respectively (Table 17). The rates in knowing the disease among the groups of professional, clerical and technical workers were as high as 98 per cent, 95.2 per cent, and 93.5 per cent respectively. The lowest rates were observed in laborers (Table 18), and farmers, $81.8 \%$ and $82.2 \%$, respectively, who may have included more illiterate people.

\section{Source of Information Concerning Tuberculosis}

"From whom or which route did you get information concerning tuberculosis?"

As a medium or source of information for tuberculosis, health center workers were playing a dominant role (35.5\% for all areas), as well as channels such as neighbors (36.8\%) and radios (11.9\%). Only 5.2 per cent was from the TB association activities, but we must know that various activities of health education, such as broadcasting, publication of a TB magazine, and news release on newspapers, have been offered by this organization. Of all channels of information, human channels comprising health center workers and neighbors turned out to be the most common source of information (72.3\%), in contrast to the non-human channels which consisted mostly of mass-media (27.3\%). The assumption that in the rural area human sources of information might have contributed to the spread of knowledge to a greater extent than in the urban areas seems corraborated by the higher "human" percentage observable in the rural areas (84.3\%) than in the urban (56.8\%). These findings, we believe, are a simple reflection of the social, economic, and cultural differences existing between the urban and rural areas of Korea.

Table 19. Number and Per Cent of Urban and Rural Respondents

\begin{tabular}{|c|c|c|c|c|c|c|}
\hline \multirow{2}{*}{$\begin{array}{l}\text { Source of } \\
\text { information }\end{array}$} & \multicolumn{2}{|c|}{ Urban } & \multicolumn{2}{|c|}{ Rural } & \multicolumn{2}{|c|}{ Total } \\
\hline & No. & $\%$ & No. & $\%$ & No. & $\%$ \\
\hline Newspaper & 52 & 14.4 & 30 & 6.5 & 82 & 9.9 \\
\hline Radio & 52 & 14.5 & 46 & 9.9 & 98 & 11.9 \\
\hline $\begin{array}{l}\text { Movie } \\
\text { TB }\end{array}$ & 31 & 8.7. & 6 & 1.2 & 37 & 4.5 \\
\hline $\begin{array}{l}\text { Association } \\
\text { Activities }\end{array}$ & 28 & 7.9 & 15 & 3.2 & 43 & 5.2 \\
\hline $\begin{array}{l}\text { Health } \\
\text { Center } \\
\text { Worker }\end{array}$ & 81 & 22.5 & 212 & 45.6 & 293 & 35.5 \\
\hline $\begin{array}{l}\text { Neighbors } \\
\text { and } \\
\text { Friends }\end{array}$ & 124 & 34.3 & 180 & 38.7 & 304 & 36.8 \\
\hline Booklets & 40 & 11.2 & 30 & 6.5 & 70 & 8.5 \\
\hline School & 56 & 15.5 & 58 & 12.5 & 114 & 13.8 \\
\hline Others & 29 & 8.1 & 17 & 3.8 & 46 & 5.6 \\
\hline I del icsp & ta) ( & & & & & \\
\hline
\end{tabular}

\section{Experience of Tuberculosis}

"Did you or do you have any pulmonary tuberculosis patients among your family members?"

Table 20 shows that 8.3 per cent of the respondents. 
have or had a tuberculosis patient among their family members. This, of course, cannot be regarded as of any statistical significance, but is valuable to estimate the proportion of people who have had a living experience with tuberculosis. Such experience is the most influencial factor for better motivation in health education

Table 20. Number and Per Cent of Urban and Rural Respondents

\begin{tabular}{c|r|r|r|r|r|r}
\hline \hline \multirow{2}{*}{$\begin{array}{c}\text { Experience of } \\
\text { tuberculosis }\end{array}$} & \multicolumn{2}{|c|}{ Urban } & \multicolumn{2}{c|}{ Rural } & \multicolumn{2}{c}{ Total } \\
\cline { 2 - 7 } & No. & $\%$ & No. & $\%$ & No. & $\%$ \\
\hline Yes & 39 & 10.8 & 29 & 6.3 & 68 & 8.3 \\
No & 322 & 89.2 & 436 & 93.7 & 758 & 91.7 \\
\hline Total & 361 & 100.0 & 465 & 100.0 & 826 & 100.0 \\
\hline
\end{tabular}

5. Mode of Transmission of Tuberculosis

a. "Do you believe that tuberculosis is inherited?"

Tuberculosis is not inherited, but is communicable. Based on this fact, the proportion of those who gave the correct answer, --"No"--, is 54.5 per cent in the urban and 51.0 per cent in the rural areas. 30.1 per cent of the respondents still held the out-dated idea that tuberculosis results from heredity which leads to the social stigma associated with the disease (Table 21). Males, younger age groups and those with high
결핵 및 호홉기질환 Vol. 18 , No. 4, October, 1971

educational background gave a higher rate of correct answers than the females, older age groups and those with low educational background (Table 22, 23, 24). We find that sales workers, laborers and housewives had higher rates of incorrect and an inadequate understanding (Table 25). With the prevailing social

Number and Per Cent of the Respondents

Table 21. Classification by Urban and Rural

\begin{tabular}{l|r|r|r|r|r|r}
\hline \hline \multirow{2}{*}{ Answer } & \multicolumn{2}{|c|}{ Urban } & \multicolumn{2}{c|}{ Rural } & \multicolumn{2}{c}{ Total } \\
\cline { 2 - 5 } \cline { 3 - 6 } & No. & $\%$ & No. & $\%$ & No. & $\%$ \\
\hline Yes & 109 & 30.2 & 140 & 30.1 & 249 & 30.1 \\
No & 213 & 59.0 & 237 & 51.0 & 450 & 54.5 \\
Don't know & 39 & 10.8 & 88 & 18.9 & 127 & 15.4 \\
\hline Total & 361 & 100.0 & 465 & 100.0 & 826 & 100.0 \\
\hline
\end{tabular}

Table 22. Classification by Sex

\begin{tabular}{l|r|r|r|r|r|r}
\hline \multirow{2}{*}{ Answer } & \multicolumn{2}{|c|}{ Male } & \multicolumn{2}{c|}{ Female } & \multicolumn{2}{c}{ Both sexes } \\
\cline { 2 - 7 } & No. & $\%$ & No. & $\%$ & No. & $\%$ \\
\hline Yes & 106 & 27.0 & 143 & 33.0 & 249 & 30.1 \\
No & 237 & 60.5 & 213 & 49.1 & 450 & 54.5 \\
Don't know & 49 & 12.5 & 78 & 17.9 & 127 & 15.4 \\
\hline Total & 392 & 100.0 & 434 & 100.0 & 826 & 100.0 \\
\hline
\end{tabular}

Toble 23. Clssification by Age Group

\begin{tabular}{|c|c|c|c|c|c|c|c|c|c|c|c|c|}
\hline \multirow{3}{*}{ Answer } & \multicolumn{12}{|c|}{ Age group } \\
\hline & \multicolumn{2}{|c|}{$20 \sim 29$} & \multicolumn{2}{|c|}{$30 \sim 39$} & \multicolumn{2}{|c|}{$40 \sim 49$} & \multicolumn{2}{|c|}{$50 \sim 59$} & \multicolumn{2}{|c|}{$60+$} & \multicolumn{2}{|c|}{ Total } \\
\hline & No. & $\%$ & No. & $\%$ & No. & $\%$ & No. & $\%$ & No. & $\%$ & No. & $\%$ \\
\hline Yes & 45 & 23.1 & 69 & 26.2 & 53 & 34.9 & 44 & 37.0 & 38 & 39.2 & 249 & 30.1 \\
\hline No & 126 & 64.6 & 159 & 60.5 & 78 & 51.3 & 53 & 44.5 & 34 & 35.1 & 450 & 54.5 \\
\hline Don't know & 24 & 12.3 & 35 & 13.3 & 21 & 13.8 & 22 & 18.5 & 25 & 25.7 & 127 & 15.4 \\
\hline Total & 195 & 100.0 & 263 & 100.0 & 152 & 100.0 & 119 & 100.0 & 97 & 100.0 & 826 & 100.0 \\
\hline
\end{tabular}

Table 24. Classification by Educational Level

\begin{tabular}{|c|c|c|c|c|c|c|c|c|c|c|c|c|c|c|}
\hline \multirow{2}{*}{ Answer } & \multicolumn{2}{|c|}{ Illiterate } & \multicolumn{2}{|c|}{$\begin{array}{c}\text { Literate no } \\
\text { schooling }\end{array}$} & \multicolumn{2}{|c|}{$\begin{array}{c}\text { Elementary } \\
\text { school }\end{array}$} & \multicolumn{2}{|c|}{$\begin{array}{l}\text { Middle } \\
\text { school }\end{array}$} & \multicolumn{2}{|c|}{ High school } & \multicolumn{2}{|c|}{ College } & \multicolumn{2}{|c|}{ Total } \\
\hline & No. & $\%$ & No. & $\%$ & No. & $\%$ & No. & $\%$ & No. & $\%$ & No. & $\%$ & No. & $\%$ \\
\hline Yes & 52 & 35.4 & 44 & 38.9 & 97 & 34.6 & 30 & 24.0 & 24 & 19.8 & 2 & 5.0 & 249 & 30.1 \\
\hline No & 44 & 29.9 & 43 & 38.1 & 142 & 50.7 & 92 & 73.6 & 93 & 76.7 & 36 & 90.0 & 450 & 54.5 \\
\hline Don't know & 51 & 34.7 & 26 & 23.0 & 41 & 14.7 & 3 & 2.4 & 4 & 3.3 & 2 & 5.0 & 127 & 15.4 \\
\hline Total & 147 & 100.0 & 113 & 100.0 & 280 & 100.0 & 125 & 100.0 & 121 & 100.0 & 40 & 100.0 & 826 & 100.0 \\
\hline
\end{tabular}


결핵 및 호흡기질환 Vol. 18, No. 4, October, 1971

Table 25. Classification by Occupation

\begin{tabular}{|c|c|c|c|c|c|c|c|c|c|c|c|c|c|c|c|c|}
\hline \multirow{2}{*}{ Answer } & \multicolumn{2}{|c|}{ Laborer } & \multicolumn{2}{|c|}{ Farmer } & \multicolumn{2}{|c|}{ Housewife } & \multicolumn{2}{|c|}{$\begin{array}{c}\text { Sales } \\
\text { worker }\end{array}$} & \multicolumn{2}{|c|}{$\begin{array}{c}\text { Technical } \\
\text { worker }\end{array}$} & \multicolumn{2}{|c|}{$\begin{array}{l}\text { Clerical } \\
\text { worker }\end{array}$} & \multicolumn{2}{|c|}{$\begin{array}{c}\begin{array}{c}\text { Professional } \\
\text { worker }\end{array} \\
\end{array}$} & \multicolumn{2}{|c|}{ Total } \\
\hline & No. & $\%$ & No. & $\%$ & No. & $\%$ & No. & $\%$ & No. & $\%$ & No. & $\%$ & No. & $\%$ & No. & $\%$ \\
\hline Yes & 74 & 36.3 & 37 & 28.2 & 85 & 30.7 & 23 & 25.0 & 5 & 16.1 & 7 & 16.7 & 18 & 36.7 & 249 & 30.1 \\
\hline No & 104 & 51.0 & 71 & 54.2 & 138 & 49.8 & 52 & 56.5 & 24 & 77.4 & 34 & 80.9 & 27 & 55.1 & 450 & 54.5 \\
\hline Don't know & 26 & 12.7 & 23 & 17.6 & 54 & 19.5 & 17 & 18.5 & 2 & 6.5 & 1 & 2.4 & 4 & 8.2 & 127 & 15.4 \\
\hline Total & 204 & 100.0 & 131 & 100.0 & 277 & 100.0 & 92 & 100.0 & 31 & 100.0 & 42 & 100.0 & 49 & 100.0 & 826 & 100.0 \\
\hline
\end{tabular}

stigma of the disease, people who have tuberculosis usually do not relate it to others. Such an attitude has also been a factor in affecting proper control of tuberculosis patients at health centers, particularly in rural areas. They hesitate to visit the health center or myon office to collect anti-tuberculosis drugs. Since they are afraid of being known as tuberculosis patients by the officials of the health center or myon office who know them, it is also not an uncommon occurrence that home visits by tuberculosis follow-up workers were refused.

b. "Do you think that tuberculosis is a communicable disease and healthy persons can be infected from a tuberculosis patient?"

Based on the fact that tuberculosis is communicable, the correct answer in this question, - "yes"-, was observed at Comparatively high rate of $76.6 \%$ of the respondents, while wrong or inadequate answers showed a rate of $23.4 \%$. There was a marked dif-
Number and Per Cent of Respondents

Table 26. Classification by Urban and Rural

\begin{tabular}{l|r|r|r|r|r|r}
\hline \multirow{2}{*}{ Answer } & \multicolumn{2}{|c|}{ Urban } & \multicolumn{2}{c|}{ Rural } & \multicolumn{2}{c}{ Total } \\
\cline { 2 - 6 } \cline { 5 - 6 } & No. & \multicolumn{1}{c}{$\%$} & No. & $\%$ & No. & $\%$ \\
\hline Yes & 304 & 84.2 & 329 & 70.8 & 633 & 76.6 \\
No & 28 & 7.8 & 60 & 12.9 & 88 & 10.7 \\
Don't know & 29 & 8.0 & 76 & 16.3 & 105 & 12.7 \\
\hline Total & 361 & 100.0 & 465 & 100.0 & 826 & 100.0 \\
\hline
\end{tabular}

Table 27. Classification by Sex

\begin{tabular}{l|r|r|r|r|r|r}
\hline \hline \multirow{2}{*}{ Answer } & \multicolumn{2}{|c|}{ Male } & \multicolumn{2}{|c|}{ Female } & \multicolumn{2}{c}{ Both sexes } \\
\cline { 2 - 5 } \cline { 5 - 6 } & No. & \multicolumn{1}{|c|}{$\%$} & No. & $\%$ & No. & $\%$ \\
\hline Yes & 314 & 80.2 & 319 & 73.5 & 633 & 76.6 \\
No & 33 & 8.3 & 55 & 12.7 & 88 & 10.7 \\
Don't know & 45 & 11.5 & 60 & 13.8 & 105 & 12.7 \\
\hline Total & 392 & 100.0 & 434 & 100.0 & 826 & 100.0 \\
\hline
\end{tabular}

Table 28. Classification by Age Group

\begin{tabular}{|c|c|c|c|c|c|c|c|c|c|c|c|c|}
\hline \multirow{3}{*}{ Answer } & \multicolumn{12}{|c|}{ Age group } \\
\hline & \multicolumn{2}{|c|}{$20 \sim 29$} & \multicolumn{2}{|c|}{$30 \sim 39$} & \multicolumn{2}{|c|}{$40 \sim 49$} & \multicolumn{2}{|c|}{$50 \sim 59$} & \multicolumn{2}{|c|}{$60+$} & \multicolumn{2}{|c|}{ Total } \\
\hline & No. & $\%$ & No. & $\%$ & No. & $\%$ & No. & $\%$ & No. & $\%$ & No. & $\%$ \\
\hline Yes & 166 & 85.2 & 231 & 87.8 & 105 & 75.8 & 81 & 68.2 & 50 & 54.9 & 633 & 76.6 \\
\hline No & 9 & 4.7 & 8 & 3.1 & 24 & 9.5 & 20 & 16.7 & 27 & 24.5 & 88 & 10.7 \\
\hline Don't know & 20 & 10.1 & 24 & 9.1 & 23 & 14.7 & 18 & 15.1 & 20 & 20.6 & 105 & 12.7 \\
\hline Total & 195 & 100.0 & 263 & 100.0 & 152 & 100.0 & 119 & 100.0 & 97 & 100.0 & 826 & 100.0 \\
\hline
\end{tabular}

Table 29. Classification by Educational Level

\begin{tabular}{|c|c|c|c|c|c|c|c|c|c|c|c|c|c|c|}
\hline \multirow{2}{*}{ Answer } & \multicolumn{2}{|c|}{ Illiterate } & \multicolumn{2}{|c|}{$\begin{array}{c}\text { Literate no } \\
\text { schooling }\end{array}$} & \multicolumn{2}{|c|}{\begin{tabular}{|c}
$\begin{array}{c}\text { Elementary } \\
\text { school }\end{array}$ \\
\end{tabular}} & \multicolumn{2}{|c|}{$\begin{array}{l}\text { Middle } \\
\text { school }\end{array}$} & \multicolumn{2}{|c|}{ High school } & \multicolumn{2}{|c|}{ College } & \multicolumn{2}{|c|}{ Total } \\
\hline & No. & $\%$ & No. & $\%$ & No. & $\%$ & No. & $\%$ & No. & $\%$ & No. & $\%$ & No. & $\%$ \\
\hline Yes & 71 & 48.3 & 67 & 59.3 & 228 & 81.6 & 112 & 89.3 & 116 & 95.5 & 39 & 97.5 & 633 & 76.6 \\
\hline No & 28 & 19.0 & 20 & 17.7 & 32 & 7.0 & 6 & 5.0 & 2 & 2.1 & - & - & 88 & 10.7 \\
\hline Don't know & 48 & 32.7 & 26 & 23.0 & 20 & 11.4 & 7 & 5.7 & 3 & 2.4 & 1 & 2.5 & 105 & 12.7 \\
\hline Total & 147 & 100.0 & 113 & 100.0 & 280 & 100.0 & 125 & 100.0 & 121 & 100.0 & 40 & 100.0 & 826 & 100.0 \\
\hline
\end{tabular}


Table 30. Classification by Occupation

\begin{tabular}{|c|c|c|c|c|c|c|c|c|c|c|c|c|c|c|c|c|}
\hline \multirow{2}{*}{ Answer } & \multicolumn{2}{|c|}{ Laborer } & \multicolumn{2}{|c|}{ Farmer } & \multicolumn{2}{|c|}{ Housewife } & \multicolumn{2}{|c|}{$\begin{array}{c}\text { Sales } \\
\text { worker }\end{array}$} & \multicolumn{2}{|c|}{$\begin{array}{c}\text { Technical } \\
\text { worker }\end{array}$} & \multicolumn{2}{|c|}{$\begin{array}{l}\text { Clerical } \\
\text { worker }\end{array}$} & \multicolumn{2}{|c|}{$\begin{array}{c}\begin{array}{c}\text { Professional } \\
\text { worker }\end{array} \\
\end{array}$} & \multicolumn{2}{|c|}{ Total } \\
\hline & No. & $\%$ & No. & $\%$ & No. & $\%$ & No. & $\%$ & No. & $\%$ & No. & $\%$ & No. & $\%$ & No: & $\%$ \\
\hline Yes & 139 & 68.1 & 93 & 71.2 & 217 & 78.3 & 69 & 75.2 & 29 & 93.6 & 41 & 97.6 & 45 & 91.8 & 133 & 76.6 \\
\hline No & 28 & 13.7 & 17 & 13.0 & 35 & 12.5 & 7 & 8.1 & - & - & - & - & 1 & 2.0 & 88 & 10.7 \\
\hline Don't know & 37 & 18.2 & 21 & 15.3 & 25 & 9.2 & 16 & 16. 7 & 2 & 6.4 & 1 & 2.4 & 3 & 6.2 & 105 & 12.7 \\
\hline Total & 204 & 100.0 & 131 & 100.0 & 277 & 100.0 & 92 & 100.0 & 31 & 100.0 & 42 & 100.0 & 49 & 100.0 & 826 & 100.0 \\
\hline
\end{tabular}

ference between the urban and the rurals; the rates of incorrect or inadequate answers were $29 \%$ and 15.8 $\%$, respectively (Table 26 ). The males who usually have greater opportunities for social activities, and younger age groups, the majority of whom have formal school education gave a higher rate of correct answers (Table 27, 28). In the illiterate and literarte without schooling, more than $40 \%$ of them was not aware of the communicable nature of the disease (Table 29). The laborers, farmers, house-wives, and sales workers had a scantier knowledge than the others (Table 30). It is also observed that some respondents answered that tuberculosis is hereditary and infectious in nature.

6. Understanding of the symptoms and signs of tuberculosis.

a. "Can yon recognize a tuberculosis patient by his appearance and symptoms?"

We cannot easily recognize one as a tuberculosis case only by observing his appearance and symptoms,
Number and Per Cent of Respondents

Table 31. Classification by Urban and Rural

\begin{tabular}{l|r|r|r|r|r|r}
\hline \multirow{2}{*}{ Answer } & \multicolumn{2}{|c|}{ Urban } & \multicolumn{2}{c|}{ Rural } & \multicolumn{2}{c}{ Total } \\
\cline { 2 - 5 } \cline { 5 - 6 } & No. & \multicolumn{1}{c|}{$\%$} & No. & $\%$ & No. & $\%$ \\
\hline Yes & 191 & 52.9 & 278 & 59.8 & 469 & 56.4 \\
No & 95 & 26.3 & 97 & 20.9 & 192 & 23.6 \\
Don't know & 75 & 20.8 & 90 & 19.3 & 165 & 20.0 \\
\hline Total & 361 & 100.0 & 465 & 100.0 & 826 & 100.0 \\
\hline
\end{tabular}

Table 32. Classification by Sex

\begin{tabular}{l|r|r|r|r|r|r}
\hline \multirow{2}{*}{ Answer } & \multicolumn{2}{|c|}{ Male } & \multicolumn{2}{|c|}{ Female } & \multicolumn{2}{c}{ Both sexes } \\
\cline { 2 - 6 } & \multicolumn{1}{|c|}{ No. } & \multicolumn{1}{c|}{$\%$} & \multicolumn{1}{c}{ No. } & $\%$ & No. & $\%$ \\
\hline Yes & 229 & 58.4 & 240 & 55.3 & 469 & 56.8 \\
No & 78 & 19.9 & 114 & 26.3 & 192 & 23.2 \\
Don't know & 85 & 21.7 & 80 & 18.4 & 165 & 20.0 \\
\hline Total & 392 & 100.0 & 434 & 100.0 & 826 & 100.0 \\
\hline
\end{tabular}

Table 33. Classification by Age Group

\begin{tabular}{|c|c|c|c|c|c|c|c|c|c|c|c|c|}
\hline \multirow{3}{*}{ Answer } & \multicolumn{12}{|c|}{ Age group } \\
\hline & \multicolumn{2}{|c|}{$20 \sim 29$} & \multicolumn{2}{|c|}{$30 \sim 39$} & \multicolumn{2}{|c|}{$40 \sim 49$} & \multicolumn{2}{|c|}{$50 \sim 59$} & \multicolumn{2}{|c|}{$60+$} & \multicolumn{2}{|c|}{ Total } \\
\hline & No. & $\%$ & No. & $\%$ & No. & $\%$ & No. & $\%$ & No. & $\%$ & No. & $\%$ \\
\hline Yes & 112 & 57.4 & 160 & 60.8 & 87 & 57.2 & 66 & 55.5 & 44 & 45.4 & 469 & 56.8 \\
\hline No & 50 & 25.6 & 54 & 20.5 & 34 & 22.4 & 26 & 21.8 & 28 & 28.9 & 192 & 23.2 \\
\hline Don't know & 33 & 37.0 & 49 & 18.7 & 31 & 20.4 & 27 & 22.7 & 25 & 25.7 & 165 & 20.0 \\
\hline Total & 195 & 100.0 & 263 & 100.0 & 152 & 100.0 & 119 & 100.0 & 97 & 100.0 & 826 & 100.0 \\
\hline
\end{tabular}

Table 34. Classification by Educational Level

\begin{tabular}{|c|c|c|c|c|c|c|c|c|c|c|c|c|c|c|}
\hline \multirow{2}{*}{ Answer } & \multicolumn{2}{|c|}{ Illiterate } & \multicolumn{2}{|c|}{$\begin{array}{l}\begin{array}{l}\text { Literate no } \\
\text { schooling }\end{array} \\
\end{array}$} & \multicolumn{2}{|c|}{$\begin{array}{c}\text { Elementary } \\
\text { school }\end{array}$} & \multicolumn{2}{|c|}{$\begin{array}{r}\text { Middle } \\
\text { school }\end{array}$} & \multicolumn{2}{|c|}{ High school } & \multicolumn{2}{|c|}{ College } & \multicolumn{2}{|c|}{ Total } \\
\hline & No. & $\%$ & No. & $\%$ & No. & $\%$ & No. & $\%$ & No. & $\%$ & No. & $\%$ & No. & $\%$ \\
\hline Yes & 71 & 48.3 & 59 & 52.2 & 176 & 62.9 & 92 & 73.6 & 67 & 55.4 & 4 & 10.0 & 469 & 56.8 \\
\hline No & 36 & 24.5 & 27 & 23.9 & 53 & 18.9 & 15 & 12.0 & 29 & 24.0 & 32 & 80.0 & 192 & 23.2 \\
\hline Don't know & 40 & 27.2 & 27 & 23.9 & 51 & 18.2 & 18 & 14.4 & 25 & 20.6 & 4 & 10.0 & 165 & 20.0 \\
\hline Total & 147 & 100.0 & 113 & 100.0 & 280 & 100.0 & 125 & 100.0 & 121 & 100.0 & 40 & 100.0 & 826 & 100.0 \\
\hline
\end{tabular}


particularly in the early stages of the disease. More than half of the respondents erroneously believe that tuberculosis : is invariably accompanied by objective symptoms. Such people may lose the opportunity of early detection and prompt treatment when they contract the disease. Not like other questions, a marked difference was not observed between the groups of different educational background, areas, ages and sexes (Table 31, 32, 33, 34).

b. "If you think that you can recognize a tuberculosis patient, what are those signs and symptoms?"

As subjective symptoms and signs with which one can recognize as a tuberculosis patient, the majority answered, "thin, pale, and cough." No difference was

Table 35. Number and Urban and Rural Per Cent of Respondents

\begin{tabular}{l|r|r|r|r|r|r}
\hline \hline \multirow{2}{*}{ Answer } & \multicolumn{2}{|c|}{ Urban } & \multicolumn{2}{c|}{ Rural } & \multicolumn{2}{c}{ Total } \\
\cline { 2 - 7 } \cline { 4 - 6 } & No. & \multicolumn{1}{c|}{$\%$} & No. & $\%$ & No. & $\%$ \\
\hline Thin \& pale & 133 & 36.8 & 181 & 38.9 & 314 & 38.0 \\
Cough & 225 & 62.3 & 279 & 60.0 & 504 & 61.0 \\
$\begin{array}{c}\text { Bloody } \\
\text { sputum }\end{array}$ & 29 & 8.3 & 46 & 9.9 & 75 & 9.1 \\
$\begin{array}{c}\text { Loss of } \\
\text { appetite }\end{array}$ & 52 & 14.4 & 107 & 23.0 & 159 & 19.2 \\
\hline $\begin{array}{c}\text { (Total res- } \\
\text { pondents) }\end{array}$ & \multicolumn{2}{|c|}{$(361)$} & \multicolumn{2}{|c|}{$(465)$} & \multicolumn{3}{|c}{ (826) } \\
\hline
\end{tabular}

observed between urban and rural areas (Table 34).

\section{Understanding the Susceptibility of Tuberculosis}

a. "Do you think that you may get tuberculosis. some day?"

Concerning the attitude of the respondents to the fact that he is also susceptible to tuberculosis, nearly half of the respondents believe that they are not susceptible to tuberculosis. Urban spondents gave 64.5 $\%$ correct answers while rural gave $52.4 \%$ (Table 36 ). The proportion of correct answers increased with higher levels of educational attainment and decreased with age. This proportion rose from $34.6 \%$ for the illiterate group to $82.5 \%$ for those with a college

\section{Number and Per Cent of Respondents}

Table 36. Classification by Urban and Rural

\begin{tabular}{l|r|r|r|r|r|r|r|r|r|r}
\hline \hline Answer & \multicolumn{2}{|c|}{ Yes } & \multicolumn{2}{|c|}{ No } & \multicolumn{2}{c|}{$\begin{array}{c}\text { Don’t } \\
\text { know }\end{array}$} & \multicolumn{2}{|c|}{ Total } \\
\cline { 2 - 6 } & \multicolumn{1}{|c|}{ No. } & $\%$ & No. & $\%$ & No. & $\%$ & No. & $\%$ \\
\hline Urban & 233 & 64.5 & 92 & 25.4 & 36 & 10.1 & 361 & $(100.0)$ \\
Rural & 244 & 52.4 & 147 & 31.6 & 74 & 16.0 & 465 & 100.0 \\
\hline Total & 477 & 57.7 & 239 & 28.9 & 110 & 13.4 & 826 & 100.0 \\
\hline
\end{tabular}

Table 37. Classification by Sex

\begin{tabular}{|c|c|c|c|c|c|c|c|c|}
\hline \multirow[t]{2}{*}{ Answer } & \multicolumn{2}{|c|}{ Yes } & \multicolumn{2}{|c|}{ No } & \multicolumn{2}{|c|}{ Don't know } & \multicolumn{2}{|c|}{ Total } \\
\hline & No. & $(\%)$ & No. & $(\%)$ & No. & $(\%)$ & No. & $(\%)$ \\
\hline Male & 258 & (65.3) & 101 & $(25.7)$ & 33 & $(9.0)$ & 392 & $(100.0)$ \\
\hline Female & 219 & $(46.0)$ & 138 & (31.7) & 77 & (22.3) & 434 & $(100.0)$ \\
\hline Both sexes & 477 & $(54.1)$ & 239 & $(28.9)$ & 110 & $(17.0)$ & 826 & $(100.0)$ \\
\hline
\end{tabular}

Table 38. Classification by Age Group

\begin{tabular}{|c|c|c|c|c|c|c|c|c|}
\hline \multirow[t]{2}{*}{ Answer } & \multicolumn{2}{|c|}{ Yes } & \multicolumn{2}{|c|}{ No } & \multicolumn{2}{|c|}{ Don't know } & \multicolumn{2}{|c|}{ Total } \\
\hline & No. & $(\%)$ & No. & $(\%)$ & No. & $(\%)$ & No. & $(\%)$ \\
\hline $20 \sim 29$ & 121 & $(62.0)$ & 50 & $(25.6)$ & 24 & (12.4) & 195 & $(100.0)$ \\
\hline $30 \sim 39$ & 173 & $(65.7)$ & 58 & $(22.0)$ & 32 & (12.3) & 263 & $(100.0)$ \\
\hline $40 \sim 49$ & 91 & $(59.8)$ & 40 & (26.3) & 21 & (13.9) & 152 & $(100.0)$ \\
\hline $50 \sim 59$ & 56 & $(47.0)$ & 46 & $(38.6)$ & 17 & (14.4) & 119 & $(100.0)$ \\
\hline $60+$ & 36 & $(37.1)$ & 45 & (46.3) & 16 & (16.6) & 97 & $(100.0)$ \\
\hline Total & 477 & (57.7) & 239 & (28.9) & 110 & (13.4) & 826 & $(100.0)$ \\
\hline
\end{tabular}


Table 39. Classification by Educational Level

\begin{tabular}{|c|c|c|c|c|c|c|c|c|}
\hline \multirow{2}{*}{ Education level } & \multicolumn{2}{|c|}{ Yes } & \multicolumn{2}{|c|}{ No } & \multicolumn{2}{|c|}{ Don't konow } & \multicolumn{2}{|c|}{ Total } \\
\hline & No. & $(\%)$ & No. & $(\%)$ & No. & $(\%)$ & No. & (\%) \\
\hline Illiteracy & 51 & (34.6) & 60 & $(40.8)$ & 36 & $(24.6)$ & 147 & $(100.0)$ \\
\hline Literacy no schooling & 53 & (46.9) & 41 & $(36.2)$ & 19 & (16.9) & 113 & $(100.0)$ \\
\hline Primary school & 163 & (58.2) & 82 & (29.3) & 35 & $(12.5)$ & 280 & $(100.0)$ \\
\hline Middle school & 81 & (64.8) & 29 & (23.2) & 15 & $(12.0)$ & 125 & $(100.0)$ \\
\hline High school & 96 & (79.3) & 20 & $(16.5)$ & 5 & $(4.2)$ & 121 & $(100.0)$ \\
\hline College & 33 & $(82.5)$ & 7 & $(17.5)$ & 0 & (0) & 40 & $(100.0)$ \\
\hline Total & 477 & (47.8) & 239 & (28.9) & 110 & (13.3) & 826 & $(100.0)$ \\
\hline
\end{tabular}

background (Table 39). Age groups of $20 \sim 29$ were $62.0 \%$ in correct answer, but it was only $37.1 \%$ in the age group of 60 and above (Table 38). Like other questions, males had a better understanding on the susceptibility of tuberculosis $(65.3 \%$ vs. $46 \%)$ (Table 37).

b. "Why do you think that you will not get tuberculosis some day?"

The reasons of 239 respondents who answered they will not get tuberculosis were analysed. More than two-thirds of them said that they will not get tuberculosis simply because they think that they are in good health and that previous $\mathrm{x}$-ray examinations were negative (Table 40). It is assumed that those who believe "tuberculosis is inherited" said they will not get tuberculosis because they never had a tuberculosis patient in their family line. Still some older people believe that tuberculosis is the disease for young people.

Table 40. Number and Per Cent of Respondent

\begin{tabular}{l|r|r}
\hline \multicolumn{1}{c|}{ Reason not susceptible } & Number & Per Cent \\
\hline Previous X-ray ex. revealed & 31 & 13.0 \\
$\quad$ negative & 139 & 58.0 \\
In good health & 28 & 11.7 \\
Old enough & 37 & 15.5 \\
No TB among family & 4 & 1.7 \\
Don't know & 239 & 100.0 \\
\hline Total &
\end{tabular}

8. Knowledge of the Method of Diagnosis for Pulmonary Tuberculosis

"What is the diagnostic measure for pulmonary tuberculosis?"

More than two-thirds of the respondents knew the diagnostic measure of tuberculosis; $59.3 \%$ in $\mathrm{x}$-ray examination and $7.9 \%$ in sputum examination. The Government has been examining yearly more than three million persons by means of $x$-ray and sputum examinations, but $32.8 \%$ of the respondents are still ignorant about the proper diagnotsic measures of tuberculosis. Since 1968, the Government has been carrying out intensive case-finding by means of sputum examination throughout the country, but it is discouraging to note that only $13.1 \%$ of the respondents mentioned sputum examination.

The percentage of the urban respondents who knew about $\mathrm{x}$-ray examination was relatively higher than that of the rural; $66.8 \%$ for the former and $53.5 \%$ for the latter. However, in sputum examination, the higher rate was observed among the rural respondents than the urban (Table 4.1). This may be attributed to the factor that more intensive sputum case-finding campaigns by tuberculosis follow-up

Table 41. Classification by Urban and Rural

\begin{tabular}{l|r|r|r|r|r|r}
\hline \hline \multirow{2}{*}{$\begin{array}{l}\text { Method of } \\
\text { diagnosis }\end{array}$} & \multicolumn{2}{|c|}{ Urban } & \multicolumn{2}{c|}{ Rural } & \multicolumn{2}{c}{ All Areas } \\
\cline { 2 - 5 } \cline { 5 - 7 } & No. & \multicolumn{1}{c}{$\%$} & No. & $\%$ & No. & $\%$ \\
\hline $\begin{array}{c}\text { X-ray exami- } \\
\text { nation }\end{array}$ & 241 & 66.8 & 249 & 53.5 & 490 & 59.3 \\
$\begin{array}{c}\text { Sputum exa- } \\
\text { mination }\end{array}$ & 21 & 5.8 & 44 & 9.2 & 65 & 7.9 \\
$\begin{array}{c}\text { Physical ex. } \\
\text { by M. D. }\end{array}$ & 40 & 11.0 & 50 & 10.8 & 90 & 10.9 \\
$\begin{array}{c}\text { Tuberculin } \\
\text { test }\end{array}$ & 7 & 2.0 & 11 & 2.4 & 18 & 2.2 \\
Don't know & 52 & 14.4 & 111 & 23.8 & 163 & 19.7 \\
\hline Total & 361 & 100.0 & 465 & 100.0 & 826 & 100.0 \\
\hline
\end{tabular}


결핵 및 호흡기질환 Vol. 18, No. 4, October, 1971

Table 42. Classification by Age Group

\begin{tabular}{|c|c|c|c|c|c|c|c|c|c|c|c|c|}
\hline \multirow{3}{*}{ Method of diagnosis } & \multicolumn{12}{|c|}{ Age group } \\
\hline & \multicolumn{2}{|c|}{$20 \sim 29$} & \multicolumn{2}{|c|}{$30 \sim 39$} & \multicolumn{2}{|c|}{$40 \sim 49$} & \multicolumn{2}{|c|}{$50 \sim 59$} & \multicolumn{2}{|c|}{$60+$} & \multicolumn{2}{|c|}{ All ages } \\
\hline & No. & $\%$ & No. & $\%$ & No. & $\%$ & No. & $\%$ & No. & $\%$ & No. & $\%$ \\
\hline $\mathrm{X}$-ray examination & 143 & 73.3 & 170 & 64.6 & 88 & 57.9 & 58 & 48.7 & 31 & 32.0 & 490 & 59.3 \\
\hline $\begin{array}{r}\text { Sputu } \\
\text { exal }\end{array}$ & 16 & 8.2 & 29 & 11.0 & 7 & 4.6 & 9 & 7.6 & 4 & 4.1 & 65 & 7.9 \\
\hline $\begin{array}{l}\text { Physical ex. by } \\
\text { M.D. }\end{array}$ & 16 & 8.2 & 22 & 8.4 & 21 & 13.8 & 13 & 10.9 & 18 & 18.6 & 90 & 10.9 \\
\hline Tuberculin test & 3 & 1.5 & 4 & 1.5 & 3 & 2.0 & 5 & 4.2 & 3 & 3.1 & 18 & 2.2 \\
\hline Don't know & 17 & 8.8 & 38 & 14.5 & 33 & 21.7 & 34 & 28.6 & 41 & 42.2 & 163 & 19.7 \\
\hline Total & 195 & 100.0 & 263 & 100.0 & 152 & 100.0 & 119 & 100.0 & 97 & 100.0 & 826 & 100.0 \\
\hline
\end{tabular}

Table 43. Classification by Educational Level

\begin{tabular}{|c|c|c|c|c|c|c|c|c|c|c|c|c|c|c|}
\hline \multirow{3}{*}{$\begin{array}{l}\text { Method of } \\
\text { diagnosis }\end{array}$} & \multicolumn{14}{|c|}{ Education level } \\
\hline & \multicolumn{2}{|c|}{ Illiterate } & \multicolumn{2}{|c|}{$\begin{array}{l}\text { Lit. no } \\
\text { school }\end{array}$} & \multicolumn{2}{|c|}{$\begin{array}{l}\text { Primary } \\
\text { school }\end{array}$} & \multicolumn{2}{|c|}{$\begin{array}{l}\text { Middle } \\
\text { school }\end{array}$} & \multicolumn{2}{|c|}{ High school } & \multicolumn{2}{|c|}{ College } & \multicolumn{2}{|c|}{ Total } \\
\hline & No. & $\%$ & No. & $\%$ & No. & $\%$ & No. & $\%$ & No. & $\%$ & No. & $\%$ & No. & $\%$ \\
\hline $\mathrm{X}$-ray examination & 51 & 34.7 & 52 & 46.0 & 169 & 60.3 & 91 & 72.8 & 94 & 77.7 & 33 & 82.5 & 490 & 59.3 \\
\hline $\begin{array}{l}\text { Sputum } \\
\text { examination }\end{array}$ & 3 & 2.0 & 3 & 2.7 & 24 & 8.6 & 14 & 11.2 & 15 & 12.4 & 6 & 15.0 & 65 & 7.9 \\
\hline $\begin{array}{l}\text { Physical ex. by } \\
\text { M.D. }\end{array}$ & 22 & 15.0 & 16 & 14.2 & 32 & 11.4 & 13 & 10.4 & 6 & 5.0 & 1 & 2.5 & 90 & 10.9 \\
\hline Tuberculin test & 7 & 4.8 & 4 & 3.5 & 3 & 1.1 & 3 & 2.4 & 1 & 0.8 & - & - & 18 & 2.2 \\
\hline Don't know & 64 & 43.5 & 38 & 33.6 & 52 & 18.6 & 4 & 3.2 & 5 & 4.1 & -1 & -1 & 163 & 19.7 \\
\hline Total & 147 & 100.0 & 113 & 100.0 & 280 & 100.0 & 125 & 100.0 & 121 & 100.0 & 40 & 100.0 & 826 & 100.0 \\
\hline
\end{tabular}

workers of health centers were carried out in rural areas than in urban areas. The proportion of those knowing $\mathrm{x}$-ray and sputum examinations is higher among the younger age group and those with higher educational level. It might reasonably be said that the rate of knowledge rose with the degree of educational background (Table 42,43).

\section{Experience of $X$-ray Examination and Attitudes toward free $\mathrm{X}$-ray examination.}

a. "Have you ever had a chest $\mathrm{x}$-ray examination?"

46. 2 per cent of the respondents had experience of $\mathrm{X}$-ray examination in the past and 53.8 per cent of them had never had any X-ray check up. The higher rate of experience in X-ray examination was observed in the urban rather than in the rural groups. (55.1\% vs. $39.4 \%$, Table 44$)$. The younger age group had a higher rate of experience than the older age group; $62.6 \%$ in age group of $20 \sim 29$, while it was only $27.8 \%$ in age group of 60 and above (Table 46). By analyzing educational levels, it was also observed, as in other questions, that the higher the educational level, the higher the rate of experience. It was $92.5 \%$

Number and Per Cent of the Respondents

Table 44. Classification by Urban and Rural

\begin{tabular}{c|r|r|r|r|r|r}
\hline \hline \multirow{2}{*}{ Answer } & \multicolumn{2}{|c|}{ Urban } & \multicolumn{2}{c|}{ Rural } & \multicolumn{2}{c}{ Total } \\
\cline { 2 - 6 } \cline { 5 - 7 } & No. & $\%$ & No. & $\%$ & No. & $\%$ \\
\hline Yes & 199 & 55.1 & 183 & 39.4 & 382 & 46.2 \\
No & 162 & 44.9 & 282 & 60.6 & 444 & 53.8 \\
\hline Total & 361 & 100.0 & 465 & 100.0 & 826 & 100.0 \\
\hline
\end{tabular}

Table 45. Classification by Sex

\begin{tabular}{c|r|r|r|r|r|r}
\hline \hline \multirow{2}{*}{ Answer } & \multicolumn{2}{|c|}{ Male } & \multicolumn{2}{c|}{ Female } & \multicolumn{2}{c}{ Both sexes } \\
\cline { 2 - 5 } \cline { 2 - 5 } & No. & $\%$ & No. & $\%$ & No. & $\%$ \\
\hline Yes & 235 & 59.9 & 147 & 33.9 & 382 & 46.2 \\
No & 157 & 40.1 & 287 & 66.1 & 444 & 53.8 \\
\hline Total & 392 & 100.0 & 434 & 100.0 & 826 & 100.0 \\
\hline
\end{tabular}


Table 46. Classification by Age Group

\begin{tabular}{|c|c|c|c|c|c|c|c|c|c|c|c|c|}
\hline \multirow{3}{*}{ Answer } & \multicolumn{12}{|c|}{ Age group } \\
\hline & \multicolumn{2}{|c|}{$20 \sim 29$} & \multicolumn{2}{|c|}{$30 \sim 39$} & \multicolumn{2}{|c|}{$40 \sim 49$} & \multicolumn{2}{|c|}{$50 \sim 59$} & \multicolumn{2}{|c|}{$60+$} & \multicolumn{2}{|c|}{ Total } \\
\hline & No. & $\%$ & No. & $\%$ & No. & $\%$ & No. & $\%$ & No. & $\%$ & No. & $\%$ \\
\hline Yes & 122 & 62.6 & 130 & 49.4 & 70 & 46.1 & 33 & 27.7 & 27 & 27.8 & 382 & 46.2 \\
\hline No & 73 & 37.4 & 133 & 50.6 & 82 & 53.9 & 86 & 72.3 & 70 & 72.2 & 444 & 53.8 \\
\hline Total & 195 & 100.0 & 263 & 100.0 & 152 & 100.0 & 119 & 100.0 & 97 & 100.0 & 826 & 100.0 \\
\hline
\end{tabular}

Table 47. Classification by Educational Level

\begin{tabular}{|c|c|c|c|c|c|c|c|c|c|c|c|c|c|c|}
\hline \multirow[b]{2}{*}{ Yes } & \multicolumn{2}{|c|}{ Illiterate } & \multicolumn{2}{|c|}{$\begin{array}{l}\text { Literate no } \\
\text { schooling }\end{array}$} & \multicolumn{2}{|c|}{$\begin{array}{c}\text { Primary } \\
\text { school }\end{array}$} & \multicolumn{2}{|c|}{$\begin{array}{l}\text { Middle } \\
\text { school }\end{array}$} & \multicolumn{2}{|c|}{ High school } & \multicolumn{2}{|c|}{ College } & \multicolumn{2}{|c|}{ Total } \\
\hline & 32 & 21.8 & 34 & 30.1 & 104 & 37.1 & 80 & 64.0 & 95 & 78.5 & 37 & 92.5 & 382 & 46.2 \\
\hline No & 115 & 78.2 & 79 & 69.9 & 176 & 62.9 & 45 & 36.0 & 26 & 21.5 & 3 & 7.5 & 444 & 53.8 \\
\hline Total & 147 & 100.0 & 113 & 100.0 & 280 & 100.0 & 125 & 100.0 & 121 & 100.0 & 40 & 100.0 & 826 & 100.0 \\
\hline
\end{tabular}

Table 48. Classification by Occupation

\begin{tabular}{|c|c|c|c|c|c|c|c|c|c|c|c|c|c|c|c|c|}
\hline \multirow{2}{*}{ Answer } & \multicolumn{2}{|c|}{ Laborer } & \multicolumn{2}{|c|}{ Farmer } & \multicolumn{2}{|c|}{ Housewife } & \multicolumn{2}{|c|}{$\begin{array}{c}\text { Sales } \\
\text { worker }\end{array}$} & \multicolumn{2}{|c|}{$\begin{array}{l}\text { Technical } \\
\text { worker }\end{array}$} & \multicolumn{2}{|c|}{$\begin{array}{l}\text { Clerical } \\
\text { worker }\end{array}$} & \multicolumn{2}{|c|}{$\begin{array}{c}\text { Professional } \\
\text { worker }\end{array}$} & \multicolumn{2}{|c|}{ Total } \\
\hline & No. & $\%$ & No. & $\%$ & No. & $\%$ & No. & $\%$ & No. & $\%$ & No. & $\%$ & No. & $\%$ & No. & $\%$ \\
\hline Yes & 91 & 44.6 & 55 & 42.0 & 84 & 30.3 & 65 & 70.7 & 22 & 71.0 & 34 & 81.0 & 31 & 63.3 & 382 & 46.0 \\
\hline No & 113 & 45.4 & 76 & 58.0 & 193 & 69.7 & 27 & 29.3 & 9 & 29.0 & 8 & 19.0 & 18 & 36.7 & 444 & 54.0 \\
\hline Total & 204 & 100.0 & 131 & 100.0 & 277 & 100.0 & 92 & 100.0 & 31 & 100.0 & 42 & 100.0 & 49 & 100.0 & 826 & 100.0 \\
\hline
\end{tabular}

Table 49. Classification by Economic Condition

\begin{tabular}{|c|c|c|c|c|c|c|c|c|}
\hline \multirow{3}{*}{ Answer } & \multicolumn{8}{|c|}{ Economic class } \\
\hline & \multicolumn{2}{|c|}{ Upper } & \multicolumn{2}{|c|}{ Middle } & \multicolumn{2}{|c|}{ Lower } & \multicolumn{2}{|c|}{ Total } \\
\hline & No. & $\%$ & No. & $\%$ & No. & $\%$ & No. & $\%$ \\
\hline Yes & 51 & 68.0 & 202 & 51.3 & 129 & 36.1 & 382 & 46.2 \\
\hline No & 24 & 32.0 & 192 & 48. 7 & 228 & 63.9 & \begin{tabular}{|l|}
9 \\
\end{tabular} 444 & 53.8 \\
\hline Total & 75 & 100.0 & 394 & 100.0 & 357 & 100.0 & \begin{tabular}{l|l|}
0 & 826
\end{tabular} & 100.0 \\
\hline
\end{tabular}

of those who had a college background, while it was only $21.8 \%$ in the illiterate group (Table 47 ). The difference of the rates between different sexes, age groups, occupations, and educational levels may have been due to the fact that males, younger age groups, clerical and professional workers and those with higher education backgrounds have more frequent opportunity to have X-ray examinations by way of physical examinations, in obligatory situations, such as applying for positions, periodical health check, schools, military service, etc. The difference of the rate was also observed by economic conditions. The rates were $68.0 \%, 51.3 \%$ and $36.1 \%$ respectively in upper, middle and lower economic classes (Table 49). By occupation, the highest rate of experience was observed in the group of clerical workers (81.0\%) and the lowest in the group of housewives (30.3\%) (Table. 48).

b. "When did you receive your last chest x-ray examination?"

Only $32.5 \%$ of the respondents have had a chest $\mathrm{x}$-ray examination within the last three years and

Number and Per Cent of the Respondents

Table 50. Classification by Urban and Rural

\begin{tabular}{c|r|r|r|r|r|r}
\hline \hline \multirow{2}{*}{$\begin{array}{c}\text { No. of years } \\
\text { after last } \\
\text { examination }\end{array}$} & \multicolumn{2}{|c|}{ Urban } & \multicolumn{2}{c|}{ Rural } & \multicolumn{2}{c}{ Total } \\
\cline { 2 - 6 } & No. & $\%$ & No. & $\%$ & No. & $\%$ \\
\hline$>2$ & 99 & 27.4 & 67 & 14.4 & 166 & 20.1 \\
$2 \sim 3$ & 20 & 5.5 & 17 & 3.7 & 37 & 4.5 \\
$3 \sim 4$ & 26 & 7.2 & 39 & 8.4 & 65 & 7.9 \\
$4+$ & 54 & 15.0 & 60 & 12.9 & 114 & 13.8 \\
$\begin{array}{c}\text { Never taken } \\
\text { x-ray ex.) }\end{array}$ & $(162)$ & $(44.9)$ & $(282)$ & $(60.6)$ & $(444)$ & $(53.7)$ \\
\hline Total & 361 & 100.0 & 465 & 100.0 & 826 & 100.0 \\
\hline
\end{tabular}


결핵 및 호흡기질환 Vol. 18, No. 4, October, 1971

Table 51. Classification by Age Group

\begin{tabular}{|c|c|c|c|c|c|c|c|c|c|c|c|c|}
\hline \multirow{3}{*}{$\begin{array}{l}\text { No. of years } \\
\text { after last } \\
\text { examination }\end{array}$} & \multicolumn{12}{|c|}{ Age group } \\
\hline & \multicolumn{2}{|c|}{$20 \sim 29$} & \multicolumn{2}{|c|}{$30 \sim 39$} & \multicolumn{2}{|c|}{$40 \sim 49$} & \multicolumn{2}{|c|}{$50 \sim 59$} & \multicolumn{2}{|c|}{$60+$} & \multicolumn{2}{|c|}{ Total } \\
\hline & No. & $\%$ & No. & $\%$ & No. & $\%$ & No. & $\%$ & No. & $\%$ & No. & $\%$ \\
\hline$>2$ & 67 & 34.4 & 59 & 22.4 & 21 & 13.8 & 10 & 8.4 & 9 & 9.3 & 166 & 20.1 \\
\hline $2 \sim 3$ & 11 & 5.6 & 16 & 6.1 & 4 & 2.6 & 3 & 2.5 & 3 & 3.1 & 37 & 4.5 \\
\hline $3 \sim 4$ & 20 & 103 & 22 & 8.4 & 10 & 6.6 & 9 & 7.6 & 4 & 4.1 & 65 & 7.9 \\
\hline $4+$ & 23 & 11.8 & 35 & 13.3 & 32 & 21.1 & 14 & 11.8 & 10 & 10.3 & 114 & 13.8 \\
\hline $\begin{array}{r}\text { Never taken } \\
\text { x-ray ex.) } \\
\end{array}$ & (74) & (37.9) & (131) & $(49.8)$ & (85) & $(55.9)$ & (83) & $(69.7)$ & (71) & $(73.2)$ & (444) & $(53.7)$ \\
\hline Total & 195 & 100.0 & 263 & 100.0 & 152 & 100.0 & 119 & 100.0 & 97 & 100.0 & 826 & 100.0 \\
\hline
\end{tabular}

Table 52. Classification by Economic Condition

\begin{tabular}{|c|c|c|c|c|c|c|c|c|}
\hline \multirow{3}{*}{$\begin{array}{l}\text { No. of years after } \\
\text { last examination }\end{array}$} & \multicolumn{8}{|c|}{ Economic condition } \\
\hline & \multicolumn{2}{|c|}{ Upper } & \multicolumn{2}{|c|}{ Middle } & \multicolumn{2}{|c|}{ Lower } & \multicolumn{2}{|c|}{ Total } \\
\hline & No. & $\%$ & No. & $\%$ & No. & $\%$ & No. & $\%$ \\
\hline$>2$ & 21 & 28.0 & 89 & 22.6 & 56 & 15.7 & 166 & 20.1 \\
\hline $2 \sim 3$ & 5 & 6.7 & 21 & 5.3 & 11 & 3.1 & 37 & 4.5 \\
\hline $3 \sim 4$ & 7 & 9.3 & 37 & 9.4 & 21 & 5.9 & 65 & 7.9 \\
\hline $4+$ & 18 & 24.0 & 45 & 11.4 & 51 & 14.3 & 114 & 13.8 \\
\hline (Never taken $x$-ray ex.) & (24) & $(32.0)$ & (202) & (51.3) & (218) & $(61.0)$ & (444) & $(53.7)$ \\
\hline Total & 75 & 100.0 & 394 & 100.0 & 357 & 100.0 & 826 & 100.0 \\
\hline
\end{tabular}

$13.8 \%$ of the respondents was for more than four years. In the urban area, the rate of those who had chest $\mathrm{x}$-ray check ups within the last 3 years was $40.1 \%$, while it was only $26.5 \%$ in the runal area (Table 50). The rate of recent $x$-ray examination decreased as age increased. The rate of those. who have taken it within the last three years was $50.3 \%$ in the age group of $20 \sim 29$, but it was only $16.5 \%$ in the age group of 60 and above (Table 51). The higher the economic condition, the higher the rate of recent $x$-ray check up; it was $44 \%, 37.3 \%$, and $34.7 \%$ in upper, middle and lower economic classes, respectively (Table 52 ).

c. "If free mobile $x$-ray examination were provided, would you accept it?"

It is encouraging to note that the majority of the respondents $(90.0 \%)$ appreciate the service of mobile $\mathrm{x}$-ray units and consider them to be a trustworthy service for health care. No marked differences were observed among urban and rural, sexes, age groups and economic classes, but the group of college background had the lowest acceptance rate (Table
$53,54,55,56)$. As a whole, $10 \%$ of the respondents refused the service. The higher the educational level and economic status the more would refuse.

\section{Number and Percent of Respondents}

Table 53. Classification by Urban and Rural

\begin{tabular}{c|r|c|r|r|r|r}
\hline \hline \multirow{2}{*}{ Answer } & \multicolumn{2}{|c|}{ Urban } & \multicolumn{2}{|c|}{ Rural } & \multicolumn{2}{|c}{ Total } \\
\cline { 2 - 6 } & No. & $\%$ & No. & $\%$ & No. & $\%$ \\
\hline Yes & 320 & $(88.6)$ & 422 & $(90.8)$ & 742 & $(90.0)$ \\
No & 41 & $(11.4)$ & 43 & $(9.2)$ & 84 & $(10.0)$ \\
\hline Total & 361 & $(100.0)$ & 465 & $(100.0)$ & 826 & $(100.0)$ \\
\hline
\end{tabular}

Table 54. Classification by Sex

\begin{tabular}{c|r|c|r|r|r|c}
\hline \hline \multirow{2}{*}{ Answer } & \multicolumn{2}{|c|}{ Male } & \multicolumn{2}{|c|}{ Female } & \multicolumn{2}{|c}{ Both sexes } \\
\cline { 2 - 7 } & No. & $\%$ & No. & $\%$ & No. & $\%$ \\
\hline Yes & 361 & $(92.1)$ & 381 & $(87.8)$ & 742 & $(89.8)$ \\
No & 31 & $(7.9)$ & 53 & $(12.2)$ & 84 & $(10.2)$ \\
\hline Total & \multicolumn{2}{|c|}{$392(100.0)$} & 434 & $(100.0)$ & 826 & $(100.0)$ \\
\hline
\end{tabular}


Table 55. Classification by Age Group

\begin{tabular}{|c|c|c|c|c|c|c|c|c|c|c|c|c|}
\hline \multirow{3}{*}{ Answer } & \multicolumn{12}{|c|}{ Age group } \\
\hline & \multicolumn{2}{|c|}{$20 \sim 29$} & \multicolumn{2}{|c|}{$30 \sim 39$} & \multicolumn{2}{|c|}{$40 \sim 49$} & \multicolumn{2}{|c|}{$50 \sim 59$} & \multicolumn{2}{|c|}{$60 \%$} & \multicolumn{2}{|c|}{ Total } \\
\hline & No. & $\%$ & No. & $\%$ & No. & $\%$ & No. & $\%$ & No. & $\%$ & No. & $\%$ \\
\hline Yes & 169 & (86.7) & 240 & 91.3 & 140 & 92.1 & 106 & 89.1 & 87 & 89.7 & 742 & 89.8 \\
\hline No & 26 & (13.3) & 23 & 8.7 & 12 & 7.9 & 13 & 10.9 & 10 & 10.3 & 84 & 10.2 \\
\hline Total & 195 & $(100.0)$ & 263 & 100.0 & 152 & 100.0 & 119 & 100.0 & 97 & 100.0 & 826 & 100.0 \\
\hline
\end{tabular}

Table 56. Classification by Educational Level

\begin{tabular}{|c|c|c|c|c|c|c|c|c|c|c|c|c|c|c|}
\hline \multirow{3}{*}{ Answer } & \multicolumn{14}{|c|}{ Education level } \\
\hline & \multicolumn{2}{|c|}{ Illiterate } & \multicolumn{2}{|c|}{$\begin{array}{l}\text { Literate no } \\
\text { schooling }\end{array}$} & \multicolumn{2}{|c|}{$\begin{array}{l}\text { Primary } \\
\text { shool }\end{array}$} & \multicolumn{2}{|c|}{$\begin{array}{c}\text { Middle } \\
\text { school }\end{array}$} & \multicolumn{2}{|c|}{ High school } & \multicolumn{2}{|c|}{ College } & \multicolumn{2}{|c|}{ Total } \\
\hline & No. & $\%$ & No. & $\%$ & No. & $\%$ & No. & $\%$ & No. & $\%$ & No. & $\%$ & No. & $\%$ \\
\hline Yes & 137 & 93.2 & 105 & 92.9 & 250 & 89.3 & 112 & 89.6 & 106 & 87.6 & 32 & 80.0 & 742 & 89.8 \\
\hline No & 10 & 6.8 & 8 & 7.1 & 30 & 10.7 & 13 & 10.4 & 15 & 12.4 & 8 & 20.0 & 84 & 10.2 \\
\hline Total & 147 & 100.0 & 113 & 100.0 & 280 & 100.0 & 125 & 100.0 & 121 & 100.0 & 40 & 100.0 & 826 & 100.0 \\
\hline
\end{tabular}

Table 57. Classification by Economic Condition

\begin{tabular}{|c|c|c|c|c|c|c|c|c|}
\hline \multirow{3}{*}{ Answer } & \multicolumn{8}{|c|}{ Economic condition } \\
\hline & \multicolumn{2}{|c|}{ Upper } & \multicolumn{2}{|c|}{ Middle } & \multicolumn{2}{|c|}{ Lower } & \multicolumn{2}{|c|}{ Total } \\
\hline & No. & $\%$ & No. & $\%$ & No. & $\%$ & No. & $\%$ \\
\hline Yes & 64 & 85.3 & 358 & 90.7 & 320 & 89.6 & 742 & 89.8 \\
\hline No & 11 & 14.7 & 36 & 9.3 & 37 & 10.4 & 84 & 10.2 \\
\hline Total & 75 & 100.0 & 394 & 100.0 & 357 & 100.0 & 826 & 100.0 \\
\hline
\end{tabular}

d. "Why would you refuse free mobile x-ray service?"

We find that more than half of those who refused believed that an $\mathrm{x}$-ray examination is not necessary simply because they are in good health. $39.3 \%$ of them rejected because they are too busy or simply

Table 57a. Number and Per cent of Urban and Rural Respondents Rejected X-ray Examination by Reason

\begin{tabular}{l|r|r|r|r|r|r}
\hline \multirow{2}{*}{$\begin{array}{c}\text { Reasons of } \\
\text { Rejection }\end{array}$} & \multicolumn{2}{|c|}{ Urban } & \multicolumn{2}{|c|}{ Rural } & \multicolumn{2}{c}{ Total } \\
\cline { 2 - 7 } \cline { 4 - 7 } & No. & $\%$ & No. & $\%$ & No. & $\%$ \\
\hline Taken recently & 10 & 23.8 & 6 & 14.3 & 16 & 19.1 \\
In good health & 15 & 35.7 & 14 & 33.3 & 29 & 34.5 \\
Harmful radiation & 2 & 4.7 & 4 & 9.5 & 6 & 7.1 \\
Too busy & 7 & 16.7 & 5 & 11.9 & 12 & 14.3 \\
Simply don't like & 5 & 11.9 & 8 & 19.1 & 13 & 15.5 \\
Others & 3 & 7.2 & 5 & 11.9 & 8 & 9.5 \\
\hline Total & 42 & 100.0 & 42 & 100.0 & 84 & 100.0 \\
\hline
\end{tabular}

don't like to take the examination. Those who refused because they expressed concern for harmful radiation were only $7.1 \%$ (Table $57 \mathrm{a}$ ). It is a common belief that radiation will shorten the life span.

\section{Experience of Sputum Examination}

"Have you ever received a sputum examination to check tuberculosis?"

In order to accelerate the discovery of infectious cases, primary sputum test as a means of case-finding

\section{Number and Percent of the Respondents}

Table 58. Classification by Urban and Rural

\begin{tabular}{c|r|r|r|r|r|r}
\hline \multirow{2}{*}{ Answer } & \multicolumn{2}{|c|}{ Urban } & \multicolumn{2}{c|}{ Rural } & \multicolumn{2}{c}{ Total } \\
\cline { 2 - 6 } \cline { 3 - 6 } & No. & \multicolumn{1}{c|}{$\%$} & No. & $\%$ & No. & $\%$ \\
\hline Yes & 50 & 13.9 & 59 & 12.7 & 109 & 13.2 \\
No & 311 & 86.1 & 406 & 87.3 & 717 & 86.8 \\
\hline Total & 361 & 100.0 & 465 & 100.0 & 826 & 100.0 \\
\hline
\end{tabular}

Table 59. Classification by Sex

\begin{tabular}{c|r|r|r|r|r|r}
\hline \hline \multirow{2}{*}{ Answer } & \multicolumn{2}{|c|}{ Male } & \multicolumn{2}{c|}{ Female } & \multicolumn{2}{c}{ Both sexes } \\
\cline { 2 - 7 } \cline { 5 - 7 } & No. & \multicolumn{1}{c}{$\%$} & No. & \multicolumn{1}{c}{$\%$} & No. & \multicolumn{1}{c}{$\%$} \\
\hline Yes & 71 & 18.1 & 48 & 11.1 & 109 & 13.2 \\
No & 321 & 81.9 & 386 & 88.9 & 717 & 86.8 \\
\hline Total & 392 & 100.0 & 434 & 100.0 & 826 & 100.0 \\
\hline
\end{tabular}


결핵 및 호흡기질환 Vol. 18, No. 4, October, 1971

Table 60. Classification by Age Group

\begin{tabular}{|c|c|c|c|c|c|c|c|c|c|c|c|c|}
\hline \multirow{3}{*}{ Answer } & \multicolumn{12}{|c|}{ Age group } \\
\hline & \multicolumn{2}{|c|}{$20 \sim 29$} & \multicolumn{2}{|c|}{$30 \sim 39$} & \multicolumn{2}{|c|}{$40 \sim 49$} & \multicolumn{2}{|c|}{$50 \sim 59$} & \multicolumn{2}{|c|}{$60+$} & \multicolumn{2}{|c|}{ Total } \\
\hline & No. & $\%$ & No. & $\%$ & No. & $\%$ & No. & $\%$ & No. & $\%$ & No. & $\%$ \\
\hline Yes & 10 & 5.1 & 22 & 8.4 & 25 & 16.4 & 17 & 14.3 & 35 & 36.1 & 109 & 13.2 \\
\hline No & 185 & 94.9 & 241 & 91.6 & 127 & 83.6 & 102 & 85.7 & 62 & 63.9 & 717 & 86.8 \\
\hline Total & 195 & 100.0 & 263 & 100.0 & 152 & 100.0 & 119 & 100.0 & 97 & 100.0 & 826 & 100.0 \\
\hline
\end{tabular}

Table 61. Clssification by Educational Level

\begin{tabular}{|c|c|c|c|c|c|c|c|c|c|c|c|c|c|c|}
\hline \multirow{2}{*}{ Answer } & \multicolumn{2}{|c|}{ Illiterate } & \multicolumn{2}{|c|}{$\begin{array}{c}\text { Literate no } \\
\text { schooling }\end{array}$} & \multicolumn{2}{|c|}{$\begin{array}{c}\text { Primary } \\
\text { school }\end{array}$} & \multicolumn{2}{|c|}{$\begin{array}{l}\text { Middle } \\
\text { school }\end{array}$} & \multicolumn{2}{|c|}{ High school } & \multicolumn{2}{|c|}{ College } & \multicolumn{2}{|c|}{ Total } \\
\hline & No. & $\%$ & No. & $\%$ & No. & $\%$ & No. & $\%$ & No. & $\%$ & No. & $\%$ & No. & $\%$ \\
\hline Yes & 17 & 11.6 & 12 & 10.6 & 39 & 16.2 & 15 & 12.0 & 20 & 16.5 & 6 & 15.0 & 109 & 13.2 \\
\hline No & 130 & 88.4 & 101 & 89.4 & 241 & 83.8 & 110 & 88.0 & 101 & 83.5 & 34 & 85.0 & 717 & 86.8 \\
\hline Total & 147 & 100.0 & 113 & 100.0 & 280 & 100.0 & 125 & 100.0 & 121 & 100.0 & 40 & 100.0 & 826 & 100.0 \\
\hline
\end{tabular}

Table 62. Classification by Occupation

\begin{tabular}{|c|c|c|c|c|c|c|c|c|c|c|c|c|c|c|c|c|}
\hline \multirow{2}{*}{ Answer } & \multicolumn{2}{|c|}{ Laborer } & \multicolumn{2}{|c|}{ Farmer } & \multicolumn{2}{|c|}{ House wife } & \multicolumn{2}{|c|}{$\begin{array}{c}\text { Sales } \\
\text { worker }\end{array}$} & \multicolumn{2}{|c|}{$\begin{array}{c}\text { Tech. } \\
\text { worker }\end{array}$} & \multicolumn{2}{|c|}{$\begin{array}{l}\text { Clerical } \\
\text { worker }\end{array}$} & \multicolumn{2}{|c|}{$\begin{array}{c}\text { Professional } \\
\text { worker }\end{array}$} & \multicolumn{2}{|c|}{ Total } \\
\hline & No. & $\%$ & No. & $\%$ & No. & $\%$ & No. & $\%$ & No. & $\%$ & No. & $\%$ & No. & $\%$ & No. & $\%$ \\
\hline Yes & 19 & 9.3 & 21 & 16.0 & 28 & 10.1 & 19 & 20.7 & 3 & 9.7 & 7 & 16. 7 & 12 & 24.5 & 109 & 13.2 \\
\hline No & 185 & 90.7 & 110 & 84.0 & 249 & 89.9 & 73 & 79.3 & 28 & 90.3 & 35 & 83.9 & 37 & 75.5 & 717 & 86.8 \\
\hline Total & 204 & 100.0 & 131 & 100.0 & 277 & 100.0 & 92 & 100.0 & 31 & 100.0 & 42 & 100.0 & 49 & 100.0 & 826 & 100.0 \\
\hline
\end{tabular}

Table 63. Classification by Economic Condition

\begin{tabular}{|c|c|c|c|c|c|c|c|c|}
\hline \multirow{3}{*}{ Answer } & \multicolumn{8}{|c|}{ Economic condition } \\
\hline & \multicolumn{2}{|c|}{ Upper } & \multicolumn{2}{|c|}{ Middle } & \multicolumn{2}{|c|}{ Lower } & \multicolumn{2}{|c|}{ Total } \\
\hline & No. & $\%$ & No. & $\%$ & No. & $\%$ & No. & $\%$ \\
\hline Yes & 13 & 17.3 & 54 & 13.7 & 42 & 11.8 & 109 & 13.2 \\
\hline No & 62 & 82.7 & 340 & 86.3 & 315 & 88.2 & 717 & 86.8 \\
\hline Total & 75 & 100.0 & 394 & 100.0 & 357 & 100.0 & 826 & 100.0 \\
\hline
\end{tabular}

was introduced by the Government in late 1967. With this means alone it was possible to discover more than 20,000 cases every year, the discovery rate being around $2 \%$. This approach is very promising because as many as 20,000 cases are discovered by lay workers in a year with rather a small expenditure. By the time of this survey, approximately 2.5 million of Korea's population were examined.

The tables show that $13.2 \%$ of age 20 and above had an experience of sputum examination to check pulmonary tuberculosis. No difference was observed among different areas, occupations, education levels and economic classes, but male and older age group had higher rates of the experience in sputum examination than the females and younger age groups. This is due to the fact that older people have more frequent respiratory symptoms than younger age groups and efforts of sputum case-finding activities were rather concentrated on older age groups. (Tables 58 63)

\section{Attitude towards the curability of tuber- culosis}

"Do you think that tuberculosis is curable?"

With the appearance of many new effective antimicrobials, tuberculosis is believed a curable disease among the public. However, the successful treatment of tuberculosis is soley dependent on the attitude and practice of regularly taking drugs under the instruction of a physician. There are many treatment failure cases due to irregular and inadequate chemotherapy without supervision of a physician.

Based on these facts, the rate of right answers to this question was $75.7 \%$ for all respondents, $85.0 \%$ in urban and $68.4 \%$ in rural (Table 64). By analysing educational levels, marked differences were observed 
between different educational backgrounds. The rate increased proportionately with education. The group

\section{Number and Per Cent of Respondents}

Table 64. Classification by Urban and Rural

\begin{tabular}{l|r|r|r|r|r|r}
\hline \multirow{2}{*}{ Answer } & \multicolumn{2}{|c|}{ Urban } & \multicolumn{2}{c|}{ Rural } & \multicolumn{2}{c}{ Total } \\
\cline { 2 - 6 } \cline { 2 - 6 } & No. & $\%$ & No. & $\%$ & No. & $\%$ \\
\hline Yes & 307 & 85.0 & 318 & 68.4 & 625 & 75.7 \\
No & 36 & 10.0 & 67 & 14.4 & 103 & 12.5 \\
Don't know & 18 & 5.0 & 80 & 17.2 & 98 & 11.8 \\
\hline Total & 361 & 100.0 & 465 & 100.0 & 826 & 100.0 \\
\hline
\end{tabular}

결핵 및 호홉기질환 Vol. 18, No. 4, October, 1971 with a college beckground was $97.5 \%$, while it was only $50.3 \%$ in the illiterate group (Table 64). The older and female groups have the higher rate of misunderstanding than the younger and male groups (Table $65 \sim 67$ ).

Table 65. Classification by Sex

\begin{tabular}{l|r|r|r|r|r|r}
\hline \hline \multirow{2}{*}{ Answer } & \multicolumn{2}{|c|}{ Male } & \multicolumn{2}{c|}{ Female } & \multicolumn{2}{c}{ Both sexes } \\
\cline { 2 - 8 } & No. & $\%$ & No. & $\%$ & No. & $\%$ \\
\hline Yes & 325 & 89.9 & 300 & 69.1 & 625 & 75.7 \\
No & 39 & 9.9 & 74 & 14.7 & 103 & 12.5 \\
Don't know & 28 & 7.2 & 70 & 16.2 & 98 & 11.8 \\
\hline Total & 392 & 100.0 & 434 & 100.0 & 826 & 100.0 \\
\hline
\end{tabular}

Table 66. Classification by Educational Level

\begin{tabular}{|c|c|c|c|c|c|c|c|c|c|c|c|c|c|c|}
\hline \multirow{2}{*}{ Answer } & \multicolumn{2}{|c|}{ Illiterate } & \multicolumn{2}{|c|}{$\begin{array}{c}\text { Literate no } \\
\text { schooling }\end{array}$} & \multicolumn{2}{|c|}{$\begin{array}{c}\text { Primary } \\
\text { school }\end{array}$} & \multicolumn{2}{|c|}{$\begin{array}{c}\text { Middle } \\
\text { school }\end{array}$} & \multicolumn{2}{|c|}{ High school } & \multicolumn{2}{|c|}{ College } & \multicolumn{2}{|c|}{ Total } \\
\hline & No. & $\%$ & No. & $\%$ & No. & $\%$ & No. & $\%$ & No. & $\%$ & No. & $\%$ & No. & $\%$ \\
\hline Yes & 74 & 50.3 & 73 & 64.6 & 207 & 74.0 & 116 & 92.8 & 116 & 95.7 & 39 & 97.5 & 625 & 75.7 \\
\hline No & 35 & 23.8 & 19 & 16.8 & 39 & 14.0 & 5 & 4.0 & 5 & 4.3 & - & - & 103 & 12.5 \\
\hline Don't know & 38 & 25.9 & 21 & 18. 6 & 34 & 12.0 & 4 & 3.2 & - & - & 1 & 2.5 & 98 & 11.8 \\
\hline Total & 147 & 100.0 & 113 & 100.0 & 280 & 100.0 & 125 & 100.0 & 121 & 100.0 & 40 & 100.0 & 826 & 100.0 \\
\hline
\end{tabular}

Table 67. Classification by Age Group

\begin{tabular}{|c|c|c|c|c|c|c|c|c|c|c|c|c|}
\hline \multirow{3}{*}{ Answer } & \multicolumn{12}{|c|}{ Age group } \\
\hline & \multicolumn{2}{|c|}{$20 \sim 29$} & \multicolumn{2}{|c|}{$30 \sim 39$} & \multicolumn{2}{|c|}{$40 \sim 49$} & \multicolumn{2}{|c|}{$50 \sim 59$} & \multicolumn{2}{|c|}{$60+$} & \multicolumn{2}{|c|}{ Total } \\
\hline & No. & $\%$ & No. & $\%$ & No. & $\%$ & No. & $\%$ & No. & $\%$ & No. & $\%$ \\
\hline Yes & 163 & 83.5 & 205 & 77.8 & 113 & 74.3 & 83 & 69.7 & 61 & 63.0 & 625 & 75.7 \\
\hline No & 14 & 7.2 & 31 & 11.7 & 18 & 11.8 & 22 & 18.5 & 18 & 18.5 & 103 & 12.5 \\
\hline Don't know & 18 & 9.3 & 27 & 10.5 & 21 & 13.9 & 14 & 11.8 & 18 & 18.5 & 98 & 11.8 \\
\hline Total & 195 & 100.0 & 263 & 100.0 & 152 & 100.0 & 119 & 100.0 & 97 & 100.0 & 826 & 100.0 \\
\hline
\end{tabular}

12. Attitude towards institutions for the treatment of tuberculosis and knowledge of free treatment service for tuberculosis

a. "Where should tuberculosis be treated?"

Over two thirds of the respondents preferred to go to a hospital for their treatment of tuberculosis, while only $14.5 \%$ would go to health center, $1.5 \%$ pharmacies, $0.9 \%$ to Chinese herb clinic and $12.0 \%$ did not know where to go (Table 68,69). Our health centers are still not trusted by the people as a place for satisfactory treatment of tuberculosis. To attract the people, a critical consideration should be given to provide kind, convenient and trustworthy service at health centers as a high authority in the eye of the general public for treatment of tuberculosis.

Number and Per cent of Respondents

Table 68. Classification by Urban and Rural

\begin{tabular}{|c|c|c|c|c|c|c|}
\hline \multirow{2}{*}{$\begin{array}{l}\text { Place for } \\
\text { treatment }\end{array}$} & \multicolumn{2}{|c|}{ Urban } & \multicolumn{2}{|c|}{ Rural } & \multicolumn{2}{|c|}{ Taotl } \\
\hline & No. & $\%$ & No. & $\%$ & No. & $\%$ \\
\hline Hospital & 267 & 74.0 & 320 & 68.8 & 587 & 71.1 \\
\hline $\begin{array}{r}\text { Chinese herb } \\
\text { dispensary }\end{array}$ & 4 & 1.1 & 3 & 0.7 & 7 & 0.9 \\
\hline Drug store & 10 & 2.8 & 2 & 0.5 & 12 & 1.5 \\
\hline $\begin{array}{l}\text { Health } \\
\text { center }\end{array}$ & 51 & 14.1 & 69 & 14.8 & 120 & 14.5 \\
\hline Don't know & 29 & 8.0 & 71 & 15.2 & 100 & 12.0 \\
\hline Total & 361 & 100.0 & 465 & 100.0 & 826 & 100.0 \\
\hline
\end{tabular}


결핵 및 호홉기질환 Vol. 18, No. 4, October, 1971

Table 69. Classification by Educational Level

\begin{tabular}{|c|c|c|c|c|c|c|c|c|c|c|c|c|c|c|}
\hline \multirow{2}{*}{$\begin{array}{l}\text { Place for } \\
\text { treatmen }\end{array}$} & \multicolumn{2}{|c|}{ Illiterate } & \multicolumn{2}{|c|}{$\begin{array}{c}\text { Literate no } \\
\text { schooling }\end{array}$} & \multicolumn{2}{|c|}{$\begin{array}{c}\text { Primary } \\
\text { school }\end{array}$} & \multicolumn{2}{|c|}{$\begin{array}{c}\text { Middle } \\
\text { school }\end{array}$} & \multicolumn{2}{|c|}{ High school } & \multicolumn{2}{|c|}{ College } & \multicolumn{2}{|c|}{ Total } \\
\hline & No. & $\%$ & No. & $\%$ & No. & $\%$ & No. & $\%$ & No. & $\%$ & No. & $\%$ & No. & $\%$ \\
\hline Hospital & 86 & 58.5 & 70 & 61.9 & 202 & 72.1 & 94. & 75.2 & 101 & 83.5 & 34 & 85.0 & 587 & 71.1 \\
\hline $\begin{array}{c}\text { Chinese herb } \\
\text { dispensary }\end{array}$ & 1 & 0.7 & 4 & 3.5 & 2 & 0.7 & - & - & - & - & - & - & 7 & 0.9 \\
\hline Drug store & 1 & 0.7 & 3 & 2.7 & 5 & 1.9 & 1 & 0.8 & 1 & 0.8 & 1 & 2.5 & 12 & 1.5 \\
\hline Health center & 14 & 9.5 & 13 & 11.5 & 39 & 13.9 & 30 & 24.0 & 19 & 15.7 & 5 & 12.5 & 120 & 14.5 \\
\hline Don't know & 45 & 30.6 & 23 & 20.4 & 32 & 11.4 & - & - & - & - & - & - & 100 & 12.0 \\
\hline Total & 1471 & 00.00 & 113 & 100.0 & 280 & 100.0 & 125 & 100.0 & 121 & 100.0 & 40 & 100.0 & 826 & 100.0 \\
\hline
\end{tabular}

b. "Do you know what institution provides free treatment for tuberculosis?"

Up to the end of 1970, a total of nearly 700,000 patients had been registered and treated with free chemotherapy by health centers. Although more than

Number and Per cent of Respondents

Table 70. Classification by Urban and Rural

\begin{tabular}{|c|c|c|c|c|c|c|}
\hline \multirow{2}{*}{ Answer } & \multicolumn{2}{|c|}{ Urban } & \multicolumn{2}{|c|}{ Rural } & \multicolumn{2}{|c|}{ Total } \\
\hline & No. & $\%$ & No. & $\%$ & No. & $\%$ \\
\hline $\begin{array}{l}\text { Health } \\
\text { center }\end{array}$ & 212 & 58.7 & 260 & 55.9 & 472 & 57.1 \\
\hline Others & 20 & 5.6 & 31 & 6.7 & 51 & 6.2 \\
\hline Don't know & 129 & 35.7 & 174 & 37.4 & 303 & 36.7 \\
\hline Total & 361 & 100.0 & 465 & 100.0 & 826 & 100.0 \\
\hline
\end{tabular}

half of the respondents referred to the health center as a free treatment institution for tuberculosis, still $36.7 \%$ of the respondents were not aware of the availability of such a service This indicated that our health education effort has not been efficient to inform them of the availability of free treatment

Table 71. Classification by Sex

\begin{tabular}{l|r|r|r|r|r|r}
\hline \hline \multirow{2}{*}{ Answer } & \multicolumn{2}{|c|}{ Male } & \multicolumn{2}{c|}{ Female } & \multicolumn{2}{c}{ Both sexes } \\
\cline { 2 - 6 } \cline { 2 - 6 } & No. & $\%$ & No. & $\%$ & No. & $\%$ \\
\hline Health & 235 & 59.9 & 237 & 54.6 & 472 & 57.1 \\
center & 31 & 8.0 & 20 & 4.6 & 51 & 6.2 \\
Others & 126 & 32.1 & 177 & 40.8 & 303 & 36.7 \\
\hline Don't know & 392 & 100.0 & 434 & 100.0 & 826 & 100.0 \\
\hline Total & & & &
\end{tabular}

Table 72. Classifications by Age Group

\begin{tabular}{|c|c|c|c|c|c|c|c|c|c|c|c|c|}
\hline \multirow{3}{*}{ Answer } & \multicolumn{12}{|c|}{ Age group } \\
\hline & \multicolumn{2}{|c|}{$20 \sim 29$} & \multicolumn{2}{|c|}{$30 \sim 39$} & \multicolumn{2}{|c|}{$40 \sim 49$} & \multicolumn{2}{|c|}{$50 \sim 59$} & \multicolumn{2}{|c|}{$6 \overline{0+}$} & \multicolumn{2}{|c|}{ Total } \\
\hline & No. & $\%$ & No. & $\%$ & No. & $\%$ & No & $\%$ & No. & $\%$ & No. & $\%$ \\
\hline Health center & 123 & 63.1 & 177 & 67.3 & 88 & 57.9 & 55 & 46.2 & 29 & 29.9 & 472 & 57.1 \\
\hline Others & 10 & 5.1 & 12 & 4.6 & 9 & 5.5 & 13 & 10.9 & 7 & 7.2 & 51 & 6.2 \\
\hline Don't know & 62 & 31.8 & 74 & 28.1 & 55 & 36.2 & 51 & 42.9 & 61 & 62.9 & 303 & 36.7 \\
\hline Total & 195 & 100.0 & 263 & 100.0 & 152 & 100.0 & 119 & 100.0 & 97 & 100.0 & 826 & 100.0 \\
\hline
\end{tabular}

Table 73. Classification by Educational Levels

\begin{tabular}{|c|c|c|c|c|c|c|c|c|c|c|c|c|c|c|}
\hline \multirow{2}{*}{ Answer } & \multicolumn{2}{|c|}{ Illiterate } & \multicolumn{2}{|c|}{$\begin{array}{l}\text { literate no } \\
\text { schooling }\end{array}$} & \multicolumn{2}{|c|}{$\begin{array}{c}\text { Primary } \\
\text { school }\end{array}$} & \multicolumn{2}{|c|}{$\begin{array}{l}\text { Middle } \\
\text { school }\end{array}$} & \multicolumn{2}{|c|}{ High school } & \multicolumn{2}{|c|}{ College } & \multicolumn{2}{|c|}{ Total } \\
\hline & No. & $\%$ & No. & $\%$ & No. & $\%$ & No. & $\%$ & No. & $\%$ & No. & $\%$ & No. & $\%$ \\
\hline Health center & 54 & 36.7 & 57 & 50.4 & 157 & 56.1 & 82 & 65.6 & 93 & 76.8 & 29 & 72.5 & 472 & 57.1 \\
\hline Others & 7 & 4.8 & 4 & 3.6 & 20 & 7.1 & 10 & 8.0 & 6 & 5.0 & 4 & 10.0 & 51 & 6.2 \\
\hline Don't know & 86 & 58.5 & 52 & 46.0 & 103 & 36.8 & 33 & 36.4 & 22 & 18.2 & 7 & 17.5 & 303 & 36.7 \\
\hline Total & 147 & 100.0 & 113 & 100.0 & 280 & 100.0 & 125 & 10.0 & 121 & 100.0 & 40 & 100.0 & 826 & 100.0 \\
\hline
\end{tabular}


Table 74. Classification by Occupation

\begin{tabular}{|c|c|c|c|c|c|c|c|c|c|c|c|c|c|c|c|c|}
\hline \multirow{2}{*}{ Answer } & \multicolumn{2}{|c|}{ Laborer } & \multicolumn{2}{|c|}{ Farmer } & \multicolumn{2}{|c|}{$\begin{array}{c}\text { House } \\
\text { wife }\end{array}$} & \multicolumn{2}{|c|}{$\begin{array}{c}\text { Sales } \\
\text { worker }\end{array}$} & \multicolumn{2}{|c|}{$\begin{array}{c}\text { Tech } \\
\text { worker }\end{array}$} & \multicolumn{2}{|c|}{$\begin{array}{c}\text { Clerical } \\
\text { worker }\end{array}$} & \multicolumn{2}{|c|}{$\begin{array}{l}\text { Professional } \\
\text { worker }\end{array}$} & \multicolumn{2}{|c|}{ Total } \\
\hline & No. & $\%$ & No. & $\%$ & No. & $\%$ & No. & $\%$ & No. & $\%$ & No. & $\%$ & No. & $\%$ & No. & $\%$ \\
\hline Health center & 110 & 53.9 & 77 & 58.8 & 165 & 59.6 & 58 & 63.0 & 18 & 58.1 & 25 & 59.5 & 19 & 38.8 & 472 & 57 \\
\hline Others & 10 & 4.9 & 6 & 4.6 & 7 & 2.5 & 8 & 8.7 & 0 & 0 & 7 & 16.7 & 13 & 26.5 & 51 & 6 \\
\hline Don't know & 84 & 41.2 & 48 & 36.6 & 105 & 37.9 & 26 & 28.3 & 13 & 41.9 & 10 & 23.8 & 17 & 34.7 & 303 & 36 \\
\hline Total & 204 & 100.0 & 131 & 100.0 & 277 & 100.0 & 92 & 100.0 & 31 & 100.0 & 42 & 100.0 & 49 & 100.0 & 826 & 100 \\
\hline
\end{tabular}

service provided by health centers.

No difference was observed in the rate between different areas, sexes and occupations, but the younger age and higher education backgroudn groups had a higher rate of information regarding free treatment service of health centers (Table 70 74)

\section{Knowledge and Attitude toward BCG Vaccination}

a. "Do you know there is a vaccine for the prevention of tuberculosis?"

The BCG vaccination program has very actively been carried out since 1967 and the coverage rate of vulnerable population reached in nearly $50 \%$ as of 1970. During the last three years $(1967 \sim 1970$ ) more than ten million children were vaccinated through the nation wide health network system. We believe that the BCG program is the most effective and cheapest tuberculosis control method in a country where the prevalence rate of the disease is high and the budget for TB control is limited.

Table 74 indicates that $72 \%$ of the respondents knew that there is a vaccine to prevent tuberculosis today, but still a considerable proportion of the rural people, (32.3\%) mostly older age groups, were not aware of the availability of the vaccine (Table 75 77). Among the housewives who usually take care of their children as much as $23.8 \%$ are ignorant about the availability of the preventive vaccination of tuberculosis (Table 79). Even the groups with educational backgrounds of high school and above, many had lack of knowledge of a vaccine to prevent tuberculosis (Table 78). The highest rate of knowledge of the vaccine among different occupation was observed in the group of clerical workers $(90.5 \%)$, the lowest was laborer and technical workers (59.3\% and 64.5\%).

\section{Number and Per Cent of Respondents}

Table 75. Classification by Rural and Urban

\begin{tabular}{|c|c|c|c|c|c|c|}
\hline \multirow{2}{*}{ Answer } & \multicolumn{2}{|c|}{ Urban } & \multicolumn{2}{|c|}{ Rural } & \multicolumn{2}{|c|}{ Total } \\
\hline & No. & $\%$ & No. & $\%$ & No. & $\%$ \\
\hline Yes & 280 & 77.6 & 315 & 67.7 & 595 & 72.0 \\
\hline No & 81 & 22.4 & 150 & 32.3 & 231 & 28.0 \\
\hline Total & 361 & 100.0 & 465 & 100.0 & 826 & 100.0 \\
\hline
\end{tabular}

Table 76. Classification by Sex

\begin{tabular}{l|r|r|r|r|r|r}
\hline \multirow{2}{*}{ Answer } & \multicolumn{2}{|c|}{ Male } & \multicolumn{2}{c|}{ Female } & \multicolumn{2}{c}{ Both sexes } \\
\cline { 2 - 6 } & \multicolumn{1}{|c|}{ No. } & $\%$ & No. & $\%$ & No. & $\%$ \\
\hline Yes & 289 & 73.7 & 306 & 70.5 & 595 & 72.0 \\
No & 103 & 26.3 & 128 & 29.5 & 231 & 28.0 \\
\hline Total & 392 & 100.0 & 434 & 100.0 & 826 & 100.0 \\
\hline
\end{tabular}

Table 77. Classification by Age Group

\begin{tabular}{|c|c|c|c|c|c|c|c|c|c|c|c|c|}
\hline \multirow{3}{*}{ Answer } & \multicolumn{12}{|c|}{ Age group } \\
\hline & \multicolumn{2}{|c|}{$20 \sim 29$} & \multicolumn{2}{|c|}{$30 \sim 39$} & \multicolumn{2}{|c|}{$40 \sim 49$} & \multicolumn{2}{|c|}{$50 \sim 59$} & \multicolumn{2}{|c|}{$60+$} & \multicolumn{2}{|c|}{ Total } \\
\hline & No. & $\%$ & No. & $\%$ & No. & $\%$ & No. & $\%$ & No. & $\%$ & No. & $\%$ \\
\hline Yes & 159 & 81.5 & 202 & 76.8 & 115 & 75.7 & 77 & 64.7 & 42 & 43.3 & 595 & 72.0 \\
\hline No & 36 & 18.5 & 61 & 23.2 & 37 & 24.3 & 42 & 35.3 & 55 & 56.7 & 231 & 28.0 \\
\hline Total & 195 & 100.0 & 263 & 100.0 & 152 & 100.0 & 119 & 100.0 & 97 & 100.0 & 826 & 100.0 \\
\hline
\end{tabular}


결핵 및 호흡기질환 Vol. 18, No. 1, October, 1971

Table 78. Classification by Educational Level

\begin{tabular}{|c|c|c|c|c|c|c|c|c|c|c|c|c|c|c|}
\hline \multirow{2}{*}{ Answer } & \multicolumn{2}{|c|}{ Illiterate } & \multicolumn{2}{|c|}{$\begin{array}{c}\text { Literate no } \\
\text { schooling }\end{array}$} & \multicolumn{2}{|c|}{ Elem. school } & \multicolumn{2}{|c|}{$\begin{array}{c}\text { Middle } \\
\text { school }\end{array}$} & \multicolumn{2}{|c|}{ High school } & \multicolumn{2}{|c|}{ College } & \multicolumn{2}{|c|}{ Total } \\
\hline & No. & $\%$ & No. & $\%$ & No. & $\%$ & No. & $\%$ & No. & $\%$ & No. & $\%$ & No. & $\%$ \\
\hline Yes & 71 & 48.3 & 71 & 62.8 & 215 & 76.8 & 99 & 79.2 & 105 & 86.8 & 34 & 85.0 & 595 & 72.0 \\
\hline No & 76 & 51.7 & 42 & 37.2 & 65 & 23.2 & 26 & 20.8 & 16 & 13.2 & 6 & 15.0 & 231 & 28.0 \\
\hline Total & 147 & 100.0 & 113 & 100.0 & 280 & 100.0 & 125 & 100.0 & 121 & 100.0 & 40 & 100.0 & 826 & 100.0 \\
\hline
\end{tabular}

Table 79. Classification by Occupation

\begin{tabular}{|c|c|c|c|c|c|c|c|c|c|c|c|c|c|c|c|c|}
\hline \multirow{2}{*}{ Answer } & \multicolumn{2}{|c|}{ Laborer } & \multicolumn{2}{|c|}{ Farmar } & \multicolumn{2}{|c|}{$\begin{array}{c}\begin{array}{c}\text { House } \\
\text { wife }\end{array} \\
\end{array}$} & \multicolumn{2}{|c|}{$\begin{array}{c}\text { Sales } \\
\text { worker }\end{array}$} & \multicolumn{2}{|c|}{$\begin{array}{c}\text { Tech } \\
\text { worker }\end{array}$} & \multicolumn{2}{|c|}{$\begin{array}{l}\text { Clerical } \\
\text { worker }\end{array}$} & \multicolumn{2}{|c|}{$\begin{array}{c}\begin{array}{c}\text { Professional } \\
\text { worker }\end{array} \\
\end{array}$} & \multicolumn{2}{|c|}{ Total } \\
\hline & No. & $\%$ & No. & $\%$ & No. & $\%$ & No. & $\%$ & No. & $\%$ & No. & $\%$ & No. & $\%$ & No. & $\%$ \\
\hline Yes & 121 & 59.3 & 93 & 71.0 & 211 & 76.2 & 75 & 81.5 & 20 & 64.5 & 38 & 90.5 & 37 & 75.5 & 595 & 72.0 \\
\hline No & 83 & 40.7 & 38 & 29.0 & 66 & 23.8 & 17 & 18.5 & 11 & 35.5 & 4 & 9.5 & 12 & 24.5 & 231 & 28.0 \\
\hline Total & 204 & 100.0 & 131 & 100.0 & 277 & 100.0 & 92 & 100.0 & 31 & 100.0 & 42 & 100.0 & 49 & 100.0 & 826 & 100.0 \\
\hline
\end{tabular}

b. "Would you accept BCG vacsination for your children to prevent tuberculosis?"

It is encouraging to note that the majority would accept BCG vaccination for their children to prevent tuberculosis. No differences were observed in the degree of BCG acceptability among different areas, sexes, age groups, educational levels, and occupations (Table 79 83). Only $7.1 \%$ of the respondents rejected BCG vaccination because of the scars and side reactions of the vaccine. To attract all the mothers,

$\underline{\text { Number and Per Cent of Respondents }}$

Table 80. Classification by Urban and Rural

\begin{tabular}{l|r|r|r|r|r|r}
\hline \multirow{2}{*}{ Answer } & \multicolumn{2}{|c|}{ Urban } & \multicolumn{2}{c|}{ Rural } & \multicolumn{2}{c}{ Total } \\
\cline { 2 - 7 } & No. & $\%$ & No. & $\%$ & No. & $\%$ \\
\hline Yes & 343 & 95.5 & 424 & 91.2 & 767 & 92.9 \\
No & 18 & 5.0 & 41 & 8.8 & 59 & 7.1 \\
\hline Total & 361 & 100.0 & 465 & 100.0 & 826 & 100.0 \\
\hline
\end{tabular}

consideration must be given to improve the technique of BCG vaccination of tuberculosis follow-up workers and the quality of vaccine itself. Although no statistical data for BCG side effects were collected, a considerable number of BCG side effects have been reported.

\section{Experience of utilizing Health Center Services}

a. "Have you ever visited health center to receive free services?"

Standardized Government health center services have been provided since 1962 beginning with the

Table 81. Classification by Sex

\begin{tabular}{c|r|r|r|r|r|r}
\hline \hline \multirow{2}{*}{ Answer } & \multicolumn{2}{|c|}{ Male } & \multicolumn{2}{|c|}{ Female } & \multicolumn{2}{c}{ Both sexes } \\
\cline { 2 - 7 } & No. & $\%$ & No. & $\%$ & No. & $\%$ \\
\hline Yes & 366 & 93.4 & 401 & 92.4 & 767 & 92.9 \\
No & 26 & 6.6 & 33 & 7.6 & 59 & 7.1 \\
\hline Total & 392 & 100.0 & 434 & 100.0 & 826 & 100.0 \\
\hline
\end{tabular}

Table 82. Classification by Age Group

\begin{tabular}{|c|c|c|c|c|c|c|c|c|c|c|c|c|}
\hline \multirow{3}{*}{ Answer } & \multicolumn{12}{|c|}{ Age group } \\
\hline & \multicolumn{2}{|c|}{$20 \sim 29$} & \multicolumn{2}{|c|}{$30 \sim 39$} & \multicolumn{2}{|c|}{$40 \sim 49$} & \multicolumn{2}{|c|}{$50 \sim 59$} & \multicolumn{2}{|c|}{$60+$} & \multicolumn{2}{|c|}{ Total } \\
\hline & No. & $\%$ & No. & $\%$ & No. & $\%$ & No. & $\%$ & No. & $\%$ & No. & $\%$ \\
\hline Yes & 175 & 89.7 & 257 & 97.7 & 140 & 92.1 & 111 & 93.3 & 84 & 86.6 & 767 & 92.9 \\
\hline No & 20 & 10.3 & 6 & 2.3 & 12 & 7.9 & 8 & 6.7 & 13 & 13.4 & 59 & 7.1 \\
\hline Total & 195 & 100.0 & 263 & 100.0 & 152 & 100.0 & 119 & 100.0 & 97 & 100.0 & 826 & 100.0 \\
\hline
\end{tabular}


Table 83. Classification by Educational Level

\begin{tabular}{|c|c|c|c|c|c|c|c|c|c|c|c|c|c|c|}
\hline \multirow{2}{*}{ Answer } & \multicolumn{2}{|c|}{ Illiterate } & \multicolumn{2}{|c|}{$\begin{array}{l}\text { Literate no } \\
\text { schooling }\end{array}$} & \multicolumn{2}{|c|}{ Elem. schoòl } & \multicolumn{2}{|c|}{$\begin{array}{l}\text { Middle } \\
\text { school }\end{array}$} & \multicolumn{2}{|c|}{ High school } & \multicolumn{2}{|c|}{ College } & \multicolumn{2}{|c|}{ Total } \\
\hline & No. & $\%$ & No. & $\%$ & No. & $\%$ & No. & $\%$ & No. & $\%$ & No. & $\%$ & No. & $\%$ \\
\hline Yes & 135 & 91.8 & 106 & 93.8 & 261 & 93.2 & 116 & 92.8 & 113 & 93.4 & 36 & 90.0 & 767 & 92.9 \\
\hline No & 12 & 8.2 & 7 & 6.2 & 19 & 6.8 & 9 & 7.2 & 8 & 6.6 & 4 & 10.0 & 59 & 7.1 \\
\hline Total & 147 & 100.0 & 113 & 100.0 & 280 & 100.0 & 125 & 100.0 & 121 & 100.0 & 40 & 100.0 & 826 & 100.0 \\
\hline
\end{tabular}

Table 84. Classification by Occupation

\begin{tabular}{|c|c|c|c|c|c|c|c|c|c|c|c|c|c|c|c|c|}
\hline \multirow{2}{*}{ Answer } & \multicolumn{2}{|c|}{ Laborer } & \multicolumn{2}{|c|}{ Farmer } & \multicolumn{2}{|c|}{ House wife } & \multicolumn{2}{|c|}{$\begin{array}{c}\begin{array}{c}\text { Sales } \\
\text { worker }\end{array} \\
\end{array}$} & \multicolumn{2}{|c|}{$\begin{array}{c}\text { Tech } \\
\text { worker }\end{array}$} & \multicolumn{2}{|c|}{$\begin{array}{l}\text { Clerical } \\
\text { worker }\end{array}$} & \multicolumn{2}{|c|}{$\begin{array}{c}\text { Professional } \\
\text { worker }\end{array}$} & \multicolumn{2}{|c|}{ Total } \\
\hline & No. & $\%$ & No. & $\%$ & No. & $\%$ & No. & $\%$ & No. & $\%$ & No. & $\%$ & No. & $\%$ & No. & $\%$ \\
\hline Yes & 181 & 88.7 & 121 & 92.4 & 256 & 92.4 & 91 & 98.1 & 31 & 100.0 & 41 & 97.6 & 46 & 93.9 & 767 & 92.9 \\
\hline No & 23 & 11.3 & 10 & 7.6 & 21 & 7.6 & 1 & 1.1 & - & - & 1 & 2.4 & 3 & 6.1 & 59 & 7.1 \\
\hline Total & 204 & 100.0 & 131 & 100.0 & 277 & 100.0 & 92 & 100.0 & 31 & 100.0 & 42 & 100.0 & 49 & 100.0 & 826 & 100.0 \\
\hline
\end{tabular}

family planning and tuberculosis control services. Table 84 shows that only $36.4 \%$ had the experience of visiting health centers to receive a certain kind of service, while two-thirds of the respondents had never visited. More urban respondents utilized the health center than the rural respondents $(42.7 \%$ vs. $31.6 \%)$. This may be due to the difficulty of transportation in rural area. The groups with an educational background of elementary, middle and high school had higher rates of utilization of health centers than those with illiterate and literate without schooling and college background (Table 85 ). In economic condition, the highest rate was observed in middle class $(40.4 \%)$ and the next $33.9 \%$ of lower class and the lowest was $28.0 \%$ of upper class (Table 84 ).

\section{Number and Per Cent of the Respondents}

Table 85. Classification by Urban and Rural

\begin{tabular}{c|r|r|r|r|r|r}
\hline \hline \multirow{2}{*}{ Answer } & \multicolumn{2}{|c|}{ Urban } & \multicolumn{2}{|c|}{ Rural } & \multicolumn{2}{c}{ Total } \\
\cline { 2 - 5 } \cline { 5 - 6 } & No. & $\%$ & No. & $\%$ & No. & $\%$ \\
\hline Yes & 154 & 42.7 & 147 & 31.6 & 301 & 36.4 \\
No & 207 & 57.3 & 318 & 68.4 & 525 & 63.6 \\
\hline Total & 361 & 100.0 & 465 & 100.0 & 826 & 100.0 \\
\hline
\end{tabular}

Table 86. Classification by Educational Level

\begin{tabular}{|c|c|c|c|c|c|c|c|c|c|c|c|c|c|c|}
\hline \multirow{2}{*}{ Answer } & \multicolumn{2}{|c|}{ Illiterate } & \multicolumn{2}{|c|}{$\begin{array}{c}\text { Literate no } \\
\text { schooling } \\
\end{array}$} & \multicolumn{2}{|c|}{$\begin{array}{c}\begin{array}{c}\text { Elementary } \\
\text { school }\end{array} \\
\end{array}$} & \multicolumn{2}{|c|}{$\begin{array}{l}\text { Middle } \\
\text { school }\end{array}$} & \multicolumn{2}{|c|}{ High school } & \multicolumn{2}{|c|}{ College } & \multicolumn{2}{|c|}{ Total } \\
\hline & No. & $\%$ & No. & $\%$ & No. & $\%$ & No. & $\%$ & No. & $\%$ & No. & $\%$ & No. & $\%$ \\
\hline Yes & 33 & 22.4 & 22 & 19.5 & 115 & 41.1 & 60 & 48.0 & 58 & 47.9 & 13 & 32.5 & 301 & 36.4 \\
\hline No & 114 & 77.6 & 91 & 80.5 & 165 & 58.9 & 65 & 52.0 & 63 & 52.1 & 27 & 67.5 & 525 & 63.6 \\
\hline Total & 147 & 100.0 & 113 & 100.0 & 280 & 100.0 & 125 & 100.0 & 121 & 100.0 & 40 & 100.0 & 826 & 100.0 \\
\hline
\end{tabular}

Table 87. Classification by Economic Condition

\begin{tabular}{|c|c|c|c|c|c|c|c|c|}
\hline \multirow{3}{*}{ Answer } & \multicolumn{8}{|c|}{ Economic condition } \\
\hline & \multicolumn{2}{|c|}{ Upper } & \multicolumn{2}{|c|}{ Middle } & \multicolumn{2}{|c|}{ Lower } & \multicolumn{2}{|c|}{ Total } \\
\hline & No. & $\%$ & No. & $\%$ & No. & $\%$ & No. & $\%$ \\
\hline Yes & 21 & 28.0 & 159 & 40.4 & 121 & 33.9 & 301 & 36.4 \\
\hline No & 54 & 72.0 & 235 & 59.6 & 236 & 66.1 & 525 & 63.6 \\
\hline Total & 75 & 100.0 & 394 & 100.0 & 357 & 100.0 & 826 & 100.0 \\
\hline
\end{tabular}

b. "What service did you receive from the health center?"

Those who have ever received free tuberculosis service from the health center was $6.4 \%$ of the respondents. The proportions of other services received were: $8.7 \%$ in "family planning, $9.8 \%$ in maternal and child health, and $11.5 \%$ in general medical care and others. By analyzing urban and rural, the 
proportions of utilizing free services of health centers were higher among the urban respondents than the rural except family planning. For the tuberculosis service, the rates of utilization were $7.2 \%$ in the urban and $5.8 \%$ in the rural (Table 88 ).

Table 88. Number and Per Cent of Urban and Rural Respondents

\begin{tabular}{|c|c|c|c|c|c|c|}
\hline \multirow{2}{*}{ Service } & \multicolumn{2}{|c|}{ Urban } & \multicolumn{2}{|c|}{ Rural } & \multicolumn{2}{|c|}{ Total } \\
\hline & No. & $\%$ & No. & $\%$ & No. & $\%$ \\
\hline Tuberculosis & 26 & 7.2 & 27 & 5.8 & 53 & 6.4 \\
\hline $\begin{array}{l}\text { Family } \\
\text { planning }\end{array}$ & 27 & 7.5 & 45 & 9.7 & 72 & 8.7 \\
\hline $\begin{array}{l}\text { Maternal } \\
\text { and child } \\
\text { health }\end{array}$ & 38 & 10.5 & 43 & 9.2 & 81 & 9.8 \\
\hline Others & 63 & 17.4 & 32 & 6.9 & 95 & 11.5 \\
\hline $\begin{array}{c}\text { (Total Res- } \\
\text { pondents) }\end{array}$ & \multicolumn{2}{|c|}{ (361) } & \multicolumn{2}{|c|}{ (465) } & \multicolumn{2}{|c|}{ (826) } \\
\hline
\end{tabular}

\section{SUMMARY}

To determine the knowledge, attitudes, and practices of Korean adult population concerning tuberculosis, a questionnaire survey for 971 selected samples was conducted during the second national tuberculosis prevalence survey in 1970. The summary of the findings are as follows:

1) Radio and newspaper communication:

Nearly half of the respondents. read newspaper and more than two-thirds of the respondents had radio broadcasts communication. (Table 8 )

2) Knowledge of the disease, "tuberculosis":

The majority of the respondents (87.8 per cent) heard of tuberculosis as a disease but still a sizable number of people are ignorant of this age-old disease. (Table 14)

3) Sources of information concerning tuberculosis:

Of all channels of information, human channels, comprising health center workers and neighbors turned out to be the most common source of information (72. 3 per cent) in contrast to the non-human channels which consisted mostly of mass media (27.3 per cent). (Table 19)

4) Understanding the mode of transmission of tuberculosis:

Nearly one-third of the respondents still hold the outdated idea that tuberculosis results from heredity and nearly one-fourth did not know communicable nature of the disease. (Table 21, 26)

5) Understanding of the symptoms and signs of tuberculosis:

More than half of the respondents erroneously believe that tuberculosis is invariably accompanied by objective symptoms, such as being thin and pale, having a cough and bloody sputum and experiencing a loss of appetite. (Table 31, 35)

6) Understanding of the susceptibility of tuberculosis:

Only half of the respondents were aware of the fact that everybody is susceptible to tuberculosis (Table 36). Those who said that they would not get tuberculosis simply thought that they were in good health or that their previous $\mathrm{X}$-ray examinations were negative (Table 40).

7) Knowledge of diagnostic measure for tuberculosis: More than half of the respondents knew of the $\mathrm{X}$-ray examination and only 7.9 per cent mentioned sputum examination as a diagnostic measure of tuberculosis. About one-third of the respondents were not aware of any kind of tuberculosis check up (Table 41).

8) Experience of $X$-ray examination:

46. 2 per cent of the respondents had experience of $\mathrm{X}$-ray examination in the past and more than half of them had never had any X-ray check up (Table 44). Only one-third of the respondents received an X-ray examination within the last three years (Table 50).

9) Attitudes toward free mobile X-ray examination:

The majority of the respondents appreciate the service of mobile X-ray units. Only 10 per cent of the respondents would refuse the service for reasons of good health, being too busy or simply don't like it (Table 53, 57).

10) Experience of sputum examination:

Only 13.2 per cent of the respondents had experience of sputum examination to check tuberculosis (Table 58).

11) Attitudes toward the curability of tuberculosis: More than two-thirds of the respondents believe that tuberculosis is curable (Table 64).

12) Attitudes toward the place of tuberculosis treatment: 
More than two-thirds of the respondents preferred to go to a hospital, while only 14.5 per cent would go to health center (Table 68).

13) Knowledge of the free treatment service of the health centers:

More than half of the respondents knew the health centers as a place for free treatment for tuberculosis (Table 70).

14) Knowledge and attitudes toward BCG vaccination:

More than two-thirds of the respondents knew that there is a vaccine to prevent tuberculosis. The majority (93 per cent) would accept BCG vaccination for their children (Table 75).

15) Experience of utilizing health center services:

Only one-third of the respondents had experience in utilizing health center services, such as, 6.4 per cent in tuberculosis, 8.7 per cent in family planning, 9.8 per cent in maternal and child health, and 11.5 per cent in others (Table 85, 88).

By analyzing different groups, in general, those who had the higher rate of incorrect and inadequate understanding toward knowledge, attitudes, and practices of tuberculosis and tuberculosis control were mostly rural people, women, illiterate or poorly educated persons, laborers, farmers, and housewives, and those at a low socio-economic class.

\section{SUGGESTIONS}

Although there was improvement in the knowledge and attitude among the people in regard to tuberculosis and its control as compared with the results of a similar survey in 1965, much still has to be done to ensure that the general public has a sound knowledge of tuberculosis.

Groups who had the higher rates of incorrect and inadequate understanding toward knowledge, attitudes, and practices on tuberculosis were mostly women, illiterate or poorly educated persons, and older age groups.

This survey suggests the need for a more intensive program to be directed towards those poorly educated people. The most striking observation is that a large percentage of the respondents did not know of the available free services that are being offered by health centers. We have shown that communication between Public Health Agencies and the public is possible within the framework of the existing mass media and organizational structures. What must be done, however, is to locate and identify new methods of presenting information to block the problems of communication "tune out," and stress educational programs on the basis of tuberculosis as a disease in order that a proper frame of reference exists for the flow of information about available services. This is of the utmost importance; since, when people have the knowledge of tuberculosis as a disease, the elements of fear, "tune out" and social stigma can negate any service project. These services are for the public and therefore must be understood by the public before they can be expected to bring about positive cooperation with the health administration.

It has been said that education is the primary and indispensable weapon in the fight to minimize tuberculosis. For more effective prevention and control, efforts must be made to develop the people's awareness of the true facts about the disease and to correct the distorted public attitude toward it, and to encourage them to take more initiative and assume greater responsibility in helping themselves, their families, and their communities solve the problem collectively. Here is where health education can provide the key to the control of tuberculosis by bringing the gaps that medical knowledge and modern control measures cannot handle effectively. In setting the health education program against tuberculosis, the following objectives should be considered:

1) To increases public knowledge of tuberculosis, its cause, mode of transmission, methods of prevention and control, approved means of treatment and care, and available services for the community.

2. To help the people realize the seriousness of tuberculosis and its far-reaching effects upon their social and economic life.

3. To foster individual and civic responsibility for the prevention and control of tuberculosis.

For a stronger tuberculosis control program, especially in educational aspects, the following 
suggestions are made:

1. The organized health education activities by health center TB workers should be intensified. The workers must be taught techniques of teaching and motivating the public.

2. The health educator of the TB Association should take an active function of community organization to stimulate the public to let them find the problem as their own. Coordination of all available resources in the community must be developed and continued.

3. Radio, newspaper and other available media must be properly utilized by the TB Association and health centers.

4. Available health center services must be known to all the public and available to them in kind, convenient and trustworthy ways. Seemingly, a large percentage of the respondents have not been utilizing health center services. A more useful approach, perhaps, is to study "non-users" and "refusers."

\section{한국성인의 結核에 관한 지식, 태도, 및 실천}

보건사회부 결핵예 방과

정

1960년 이래 적극적으로 전개한 정부 및 대한결핵협회 의 보건교육사업 평가를 위하여 1971년 실시한 第2次 결핵실태조사와 병행하여 전국 86 개지역에서 971 명의

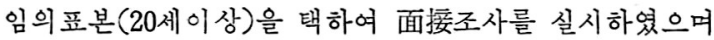
분석된 결과는 다음과 같다.

1) 신문 구독비율은 $43.8 \%$, 라더오 청취비율은 $77 \%$ 였 다.

2) 結核이라는 병을 알고 있는 사람은 소體의 $88 \%$ 였다.

3 ) 結核에 관한 지식을 대부분 $(73 \%)$ 이 보건소 결핵관 리요원 혹은 이웃을 통하여 얻었으며 mass media를 통한 지식 획득비율은 $27 \%$ 에 불과하였다.

4) 結核을 유전병으로 믿고있는 사람은 소스.6口豊의 $30 \%$ 였다.

5) 結核환자는 뚜렷한 증세(창백, 기침, 혈담)가 있다고

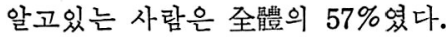

6) 自己는 건강하기 때문에 결핵에 이환되기 않을 것이 라고 믿고있는 사람은 속體의 $58 \%$ 였다.

7) 結核診斷 방법으로 $\mathrm{X}$ 선검사를 알고있는 비율은 $59 \%$ 였고 객담검사는 불과 $7.9 \%$ 였다.

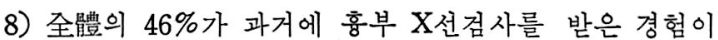

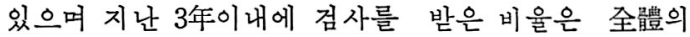

$33 \%$ 였다.

9) 대다수 $(90 \%)$ 가 무료 $X$ 선검진에 응하겠다고 하였으 며 나머지 $10 \%$ 는 필요를 느끼지 않는다는 이유로 거 절하였다.

10) 결핵진단을 위한 객담검사를 받은 경험이 있는 사 람은 소體의 $13 \%$ 였다.

11) 결핵은 고칠수 있다고 믿는 사람의 비율은 $76 \%$ 였다.

12) 대다수 $(71 \%)$ 가 결핵치료는 병원에서 받아야 한다 는 것이며 보건소를 지적한 비율은 $15 \%$ 에 지나지 않 았다.

13) 결핵 無料치료 기관으로서 보건소를 모르고 있는 사 람의 비율은 $37 \%$ 였다.

14) 대다수 $(72 \%)$ 가 결핵예방주사를 알고 있으며 어린

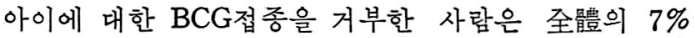
였다.

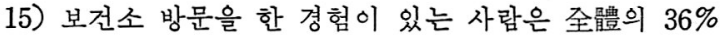
였고 결핵치료 가족계획 및 모자보건 상담이 주 이 였다.

地域, 年令, 姓, 教育, 職業, 經濟狀况별로 분석 결 과 공통적으로 농촌, 年老層, 女子, 敉育水準이 낮은 層에 서 윤곡된 지식과 태도를 가지고 있었다. 